\title{
Calidad ambiental y crecimiento económico: análisis dinámico para América Latina y el Caribe
}

\author{
Recibido: 19 de abril de 2021 - Aceptado: 23 de julio de 2021
}

Doi: https://doi.org/10.12804/revistas.urosario.edu.co/economia/a.10514

\author{
Martín Olivera* \\ Verónica Segarra ${ }^{\dagger}$
}

\section{Resumen}

Este trabajo estudia la relación dinámica entre las emisiones de dióxido de carbono y el crecimiento económico para un grupo de países de América Latina y el Caribe durante el periodo de 1951-2014. A partir del uso de regímenes económicos y simbolización de series temporales, se introduce una métrica y a través del análisis de clusters se encuentran dos grupos diferenciados en términos de dinámica de emisiones y crecimiento. Se analiza la existencia de relaciones bidireccionales tanto de corto como de largo plazo, encontrando que la relación difiere según los grupos y los métodos de estimación.

Palabras clave: crecimiento económico; emisiones de $\mathrm{CO}_{2}$; dinámica de regímenes; causalidad; América Latina.

Clasificación JEL: C36, O44, O47.

* Grupo de Investigación en Dinámica Económica, Departamento de Métodos Cuantitativos, Facultad de Ciencias Económicas y de Administración, Universidad de la República (UdelaR), Uruguay. oRciD: https://orcid.org/0000-0002-9893-3233

+ Grupo de Investigación en Dinámica Económica, Departamento de Métodos Cuantitativos, Facultad de Ciencias Económicas y de Administración, Universidad de la República (UdelaR), Uruguay. Autora de correspondencia. Dirección: Eduardo Acevedo 1139, C.P. 11200-Montevideo, Uruguay. Teléfono: (598) 24102564 int. 117. Email: vsegarra@ ccee.edu.uy. ORCID: https://orcid.org/0000-0003-0436-3303

Para citar este artículo: Olivera, M., \& Segarra, V. (2021). Calidad ambiental y crecimiento económico: análisis dinámico para América Latina y el Caribe. Revista de Economía del Rosario, 24(2), 1-40. https://doi.org/10.12804/revistas.urosario.edu.co/economia/a.10514 


\title{
Environmental Quality and Economic Growth: Dynamic Analysis for Latin America and the Caribbean
}

\begin{abstract}
This paper presents the study of the dynamic relationship between carbon dioxide emissions and economic growth for a group of Latin America and Caribbean countries during 1951-2014. From the use of economic regimes and time series symbolization, a metric was introduced. Through cluster analysis, two distinct groups emerged in terms of their emissions and growth dynamics. Analyzing the existence of bidirectional relationships both in the short and long term, it was found that the relationship differs according to the groups and estimation methods.
\end{abstract}

Keywords: Economic growth; carbon dioxide emissions; regimes dynamics; causality; Latin America.

JEL classification: C36, O44, O47.

\section{Qualidade ambiental e crescimento econômico: análise dinâmica para a América Latina e o Caribe}

\section{Resumo}

Este artigo estuda a relação dinâmica entre as emissões de dióxido de carbono e o crescimento econômico de um grupo de países da América Latina e do Caribe durante o período 1951-2014. A partir da utilização de regimes econômicos e da simbolização de séries temporais, uma métrica é introduzida e por meio da análise de clusters, dois grupos diferentes são encontrados em termos de suas emissões e dinâmica de crescimento. É analisada a existência de relações bidirecionais de curto e longo prazo, constatando que a relação difere de acordo com os grupos e os métodos de estimação.

Palavras-chave: crescimento econômico; emissões de $\mathrm{CO}_{2}$; dinâmica de regime; causalidade; América Latina.

Classificação JEL: C36, O44, O47. 


\section{Introducción}

En los últimos años se observa un considerable esfuerzo por entender la relación entre las emisiones de dióxido de carbono $\left(\mathrm{CO}_{2}\right)$ y el crecimiento económico. La emisión de $\mathrm{CO}_{2}$ tiene un papel central en el debate actual acerca del desarrollo sustentable, directamente relacionado con el consumo de energía (Nguyen \& Kakinaka, 2018; De Souza et al., 2018); y a su vez, el crecimiento económico se relaciona positivamente con un mayor nivel de consumo de energía. Por otro lado, es razonable pensar que el uso eficiente de los recursos energéticos requiera de un mayor crecimiento económico (Koengkan \& Fuinhas, 2020; Rodríguez-Caballero \& Ventosa-Santaularia, 2017; Antonakakis et al., 2017; Ito, 2017). La literatura empírica evidencia que ambos escenarios son posibles, pues algunos trabajos sugieren que las emisiones de $\mathrm{CO}_{2}$ se relacionan positivamente con el crecimiento económico, mientras que otros encuentran que el crecimiento y las emisiones de $\mathrm{CO}_{2}$ se relacionan negativamente. Para el caso particular de América Latina, existe demostración empírica a favor de la degradación ambiental a lo largo del periodo de crecimiento económico desde la década de 1940 (Sunkel \& Gligo, 1980; Gligo 1993). Además, diversos trabajos encuentran relaciones empíricas en esta línea para distintos países y periodos de tiempo (Atta Mills et al., 2020; Gardiner \& Hajek, 2020; Antonakakis et al., 2017). No solo se encuentra evidencia de las relaciones ambientales a partir del consumo de energía, emisiones de gases o crecimiento económico, sino que otros factores - geográficos, sociales, económicos- también son tenidos en cuenta en la literatura aplicando distintas metodologías en la búsqueda de relaciones de equilibrio (Amin et al., 2020; Munir et al., 2020; Vujovic et al., 2018; Chang et al., 2019; Lean \& Smyth, 2010).

Según la hipótesis de la curva ambiental de Kuznets (ЕКC por su nombre en inglés, Environmental Kuznets Curve) a medida que aumenta la producción, las emisiones de $\mathrm{CO}_{2}$ incrementan hasta un cierto nivel de producción (turning point), a partir del cual las emisiones comienzan a disminuir. Según Angulo (2010), la mayor parte de la población mundial se encuentra en el tramo creciente de la curva. Por tanto, debe existir un gran esfuerzo por parte de las autoridades de las naciones a fin de diseñar políticas económicas que mitiguen el impacto ambiental del crecimiento económico. El estudio de Grossman y Krueger (1991) surge como pionero en trabajos que buscan la validación de la hipótesis de Kuznets y, posteriormente hay trabajos que verifican esta relación (Galeotti et al., 2009; Saboori et al., 2012; Shahbaz et al., 2013; Acheampong, 2018; Munir et al., 2020; Al Mamun, 2014; Sánchez 
\& Caballero, 2019; Zambrano-Monserrate et al., 2016), mientras que otros verifican la existencia de una relación únicamente en un sentido (creciente) entre las emisiones de $\mathrm{CO}_{2}$ y el crecimiento de las economías (Azam, 2016; Antonakakis et al., 2017; Wawrzyniak \& Doryn, 2020).

La relación entre las emisiones de $\mathrm{CO}_{2}$ y el crecimiento económico ha sido estudiada utilizando distintas metodologías, diferentes países y periodos de tiempo. Estudios recientes exploran esta relación para un solo país o varios países. En particular, algunas investigaciones emplean métodos jerárquicos entre los que se pueden destacar Kantar y Keskin (2013), Deviren y Deviren (2016), Kantar et al. (2016), Kantar et al. (2019) y Souza Mendonca et al. (2020), quienes estudian grupos de países a partir de sus diferentes estructuras y comportamientos; dichos estudios se asocian fuertemente con metodologías de análisis de redes sociales y suelen encontrar relaciones que se ven explicadas por factores geográficos o factores relacionados al grado de desarrollo de las economías.

En general, los estudios que centran su análisis en un conjunto de países suponen la existencia de un comportamiento homogéneo entre las unidades del panel. Esto no parece ser plausible a la hora de observar la evidencia disponible. De esta forma, este trabajo busca realizar aportes en esa dirección, analizando las conductas de los países considerados en el panel con un enfoque dinámico, sin considerar un único modelo para todos ellos. Mediante técnicas no paramétricas, se analizará la existencia de grupos con estructuras y comportamientos similares en su dinámica, en términos de crecimiento económico y emisiones de $\mathrm{CO}_{2}$, en función de su comportamiento dinámico desde un enfoque principalmente cualitativo. Posteriormente y de acuerdo con los resultados obtenidos se estima desde un enfoque cuantitativo la relación existente entre las emisiones de $\mathrm{CO}_{2}$ y el crecimiento económico.

De ese modo, lo que resta del trabajo se organiza de la siguiente manera: la segunda sección presenta la revisión de literatura para América Latina y el Caribe; la tercera sección anuncia los datos a utilizar, la metodología empleada para la simbolización de las series, las técnicas de agrupamiento utilizadas, y la propuesta econométrica para la estimación. Por último, en la cuarta sección se exponen las consideraciones finales.

\section{Revisión de la literatura para América Latina y el Caribe}

Los estudios para América Latina y el Caribe son numerosos. Muchas investigaciones analizan la relación existente entre el crecimiento económico y el consumo de energía (Antonakakis et al., 2017; Koengkan \& Fuinhas, 2020; 
Rodríguez-Caballero \& Ventosa-Santaularia, 2017; Nguyen \& Kakinaka, 2018; Ben Mbarek et al., 2018) donde se encuentran resultados en diversos sentidos, tanto una relación causal desde el consumo de energía hacia el crecimiento y, desde el crecimiento al consumo energético, así como también en ambas direcciones. Un punto en común es la metodología, en la cual los vectores autorregresivos para paneles, los modelos vectoriales de corrección del error y la causalidad de Granger fueron las principales vías para la obtención de resultados.

Son diversos los antecedentes relevantes para la región, en cuanto al estudio del medio ambiente determinado a partir de las emisiones de dióxido de carbono, conjuntamente con el crecimiento económico. En esta línea, distintos estudios encuentran una relación causal en ambos sentidos entre el crecimiento económico y las emisiones de $\mathrm{CO}_{2}$, tanto en estudios de caso como para paneles de países (Salazar-Nuñez et al., 2020; Ben Jelbi et al., 2019; Balado-Naves et al., 2018; Apergis \& Payne, 2015, 2014, 2009; Al Mulali et al., 2015; Pao \& Tsai, 2011). Sin embargo, otros autores se contraponen a estas ideas y plantean que la relación causal entre las dos variables es unívoca, por un lado, desde las emisiones de $\mathrm{CO}_{2}$ hacia el crecimiento económico (Agbanike et al., 2019; Zambrano-Monserrate et al., 2018 ) y por otro lado, desde el crecimiento de las economías hacia las emisiones de dióxido de carbono (Al Mulali, 2014; Gómez \& Rodríguez, 2016; Dutta \& Das, 2016; Ozturk, 2017; Ben Jelbi et al., 2019; Zafar et al., 2019; Patiño et al., 2019). En estos casos, nuevamente las metodologías implementadas siguen una línea común. Entre los principales métodos se destacan el procedimiento de cointegración de Johansen (VECM) y test de causalidad en el sentido de Granger. Sin embargo, adicionalmente se encuentran estudios que optan por la utilización del método de mínimos cuadrados dinámicos (DOLS) o por la utilización de modelos autorregresivos con retardos distribuidos (ARDL). En cuanto a los periodos considerados, se encuentra también cierto grado de similitud debido a la disponibilidad de datos de las diversas fuentes que recogen información de esta índole. Así, en general los periodos considerados no superan los 40 años, y no exceden al año 2014. Debido a lo anterior, debe mantenerse cierto nivel de cautela a la hora de realizar conclusiones o interpretaciones apresuradas, dado que por lo regular, los principales antecedentes no permiten recoger efectos más contemporáneos a los considerados en un fenómeno tan dinámico como la degradación ambiental. Las tablas 1 y 2 presentan un resumen de los estudios realizados para América Latina y el Caribe, o en su defecto, que contengan países de la región. 
Tabla 1. Resumen de literatura para América Latina y el Caribe. Estudios de caso

\begin{tabular}{|c|c|c|c|c|c|}
\hline Trabajo & $\begin{array}{l}\text { Periodo } \\
\text { analizado }\end{array}$ & País & Variables & Metodología & Conclusiones \\
\hline $\begin{array}{l}\text { Agbanike } \\
\text { et al. } \\
(2019)\end{array}$ & $1971-2013$ & Venezuela & $\begin{array}{l}\text { Uso de energía, } \\
\text { emisiones de } \mathrm{CO}_{2^{\prime}} \\
\text { PIB pc real, precio } \\
\text { del petróleo, } \\
\text { gasto del Gobierno } \\
\text { general. }\end{array}$ & $\begin{array}{l}\text { ARDL, UECM, } \\
\text { causalidad de } \\
\text { Granger (IAA). }\end{array}$ & $\mathrm{CO}_{2} \rightarrow \mathrm{Y}$ \\
\hline $\begin{array}{l}\text { Patiño et al. } \\
\text { (2019) }\end{array}$ & 1971-2011 & Colombia & $\begin{array}{l}\text { Emisiones de } \mathrm{CO}_{2} \mathrm{pc} \text {, } \\
\text { energía primaria pc, } \\
\text { PIB pc, entre otras. }\end{array}$ & MCo dinámico. & $\begin{array}{l}\mathrm{Y}+\mathrm{CO}_{2} \\
\text { Se verifica EKC }\end{array}$ \\
\hline $\begin{array}{l}\text { Ben Jebli y } \\
\text { Ben Youssef } \\
\text { (2016) }\end{array}$ & $1980-2013$ & Brasil & $\begin{array}{l}\text { Consumo de com- } \\
\text { bustible, valor agre- } \\
\text { gado agrícola, emi- } \\
\text { siones de } \mathrm{CO}_{2} \text {, PIB. }\end{array}$ & $\begin{array}{l}\text { ARDL, causalidad } \\
\text { de Granger. }\end{array}$ & $\mathrm{CO}_{2} \leftrightarrow \mathrm{Y}$ \\
\hline $\begin{array}{l}\text { Gómez y } \\
\text { Rodríguez } \\
\text { (2016) }\end{array}$ & 1971-2011 & México & $\begin{array}{l}\text { PIB, emisiones } \\
\text { de } \mathrm{CO}_{2} \text {, apertura } \\
\text { comercial. }\end{array}$ & $\begin{array}{l}\text { Cointegración } \\
\text { (vECM), causali- } \\
\text { dad de Granger. }\end{array}$ & $\mathrm{Y} \rightarrow \mathrm{CO}_{2}$ \\
\hline $\begin{array}{l}\text { Pao y Tsai } \\
\text { (2011) }\end{array}$ & $1980-2007$ & Brasil & $\begin{array}{l}\text { Emisiones de } \mathrm{CO}_{2} \text {, } \\
\text { PIB real, consumo } \\
\text { de energía. }\end{array}$ & $\begin{array}{l}\text { Cointegración } \\
\text { Johansen, VECM, } \\
\text { causalidad de } \\
\text { Granger. }\end{array}$ & $\mathrm{Y} \leftrightarrow \mathrm{CO}_{2}$ \\
\hline
\end{tabular}

Fuente: elaboración propia.

Tabla 2. Resumen de literatura para América Latina y el Caribe. Estudios para paneles

\begin{tabular}{|c|c|c|c|c|c|}
\hline Trabajo & $\begin{array}{c}\text { Periodo } \\
\text { analizado }\end{array}$ & Países & Variables & Metodología & Conclusiones \\
\hline $\begin{array}{l}\text { Koengkan } \\
\text { y Fuinhas } \\
(2020)\end{array}$ & $1980-2014$ & Mercosur & $\begin{array}{l}\text { PIB, consumo } \\
\text { de energía, } \\
\text { índice KoF de } \\
\text { globalización. }\end{array}$ & $\begin{array}{l}\text { PVAR, } \\
\text { causalidad de } \\
\text { Granger }\end{array}$ & $\mathrm{CE} \rightarrow \mathrm{Y}$ \\
\hline $\begin{array}{l}\text { Wawr- } \\
\text { zyniak y } \\
\text { Doryn } \\
(2020)\end{array}$ & 1995-2014 & $\begin{array}{l}93 \text { países } \\
\text { emergentes } \\
\text { (incluye ALC) }\end{array}$ & $\begin{array}{l}\text { Emisiones de } \mathrm{CO}_{2} \text {, } \\
\text { PIB, consumo de } \\
\text { energía, IED. }\end{array}$ & Estimador GMM. & (1) \\
\hline $\begin{array}{l}\text { Salazar- } \\
\text { Nuñez et al. } \\
(2020)\end{array}$ & 1980-2014 & $\begin{array}{l}79 \text { países } \\
\text { (incluido ALC) } \\
\text { agrupados } \\
\text { por ingreso }\end{array}$ & $\begin{array}{l}\text { Consumo prima- } \\
\text { rio de energía, PIB, } \\
\text { emisiones de } \mathrm{CO}_{2} \text {. }\end{array}$ & $\begin{array}{l}\text { Cointegración } \\
\text { para datos de } \\
\text { panel (Johan- } \\
\text { sen-vECM), } \\
\text { causalidad de } \\
\text { Granger. }\end{array}$ & $\mathrm{Y} \leftrightarrow \mathrm{CO}_{2}$ \\
\hline
\end{tabular}




\begin{tabular}{|c|c|c|c|c|c|}
\hline Trabajo & $\begin{array}{l}\text { Periodo } \\
\text { analizado }\end{array}$ & Países & Variables & Metodología & Conclusiones \\
\hline $\begin{array}{l}\text { Nguyen y } \\
\text { Kakinaka } \\
(2018)\end{array}$ & 1990-2013 & $\begin{array}{l}107 \text { países (in- } \\
\text { cluido ALC) }\end{array}$ & $\begin{array}{l}\text { Consumo de } \\
\text { energía, PIB, } \\
\text { emisiones de } \\
\mathrm{CO}_{2} \text { precio del } \\
\text { petróleo. }\end{array}$ & $\begin{array}{l}\text { Cointegración } \\
\text { para paneles } \\
\text { dinámicos } \\
\text { (Pedroni-Kao) }\end{array}$ & $\begin{array}{l}\mathrm{CER}+\mathrm{CO}_{2} \\
\mathrm{CER}-\mathrm{Y} \text { (bajo } \\
\text { ingreso) } \\
\mathrm{CER}-\mathrm{CO}_{2} \\
\mathrm{CER}+\mathrm{Y} \text { (alto } \\
\text { ingreso) }\end{array}$ \\
\hline $\begin{array}{l}\text { Sánchez y } \\
\text { Caballero } \\
\text { (2019) }\end{array}$ & 1980-2015 & $\begin{array}{l}\text { América Lati- } \\
\text { na y el Caribe }\end{array}$ & $\begin{array}{l}\text { Consumo de } \\
\text { energía, ingreso } \\
\text { per cápita. }\end{array}$ & $\begin{array}{l}\text { Cointegración } \\
\text { para paneles de } \\
\text { Pedroni, FMOLs. }\end{array}$ & $\begin{array}{l}\text { EKC } \\
(2)\end{array}$ \\
\hline $\begin{array}{l}\text { Zafar et al. } \\
\text { (2019) }\end{array}$ & $1990-2015$ & $\begin{array}{l}\text { Brasil, Colom- } \\
\text { bia, México, } \\
\text { Perú y otros } \\
14 \text { países } \\
\text { emergentes }\end{array}$ & $\begin{array}{l}\text { Consumo } \\
\text { de energía, } \\
\text { PIB, apertura } \\
\text { comercial. }\end{array}$ & $\begin{array}{l}\text { Causalidad de } \\
\text { Granger - vECM }\end{array}$ & $\mathrm{Y} \rightarrow \mathrm{CO}_{2}$ \\
\hline $\begin{array}{l}\text { Ben Jebii } \\
\text { et al. (2019) }\end{array}$ & $1995-2010$ & $\begin{array}{l}22 \text { países de } \\
\text { ALC }\end{array}$ & $\begin{array}{l}\text { Emisiones de } \\
\mathrm{CO}_{2} \text {, PIB, arribos, } \\
\text { apertura } \\
\text { comercial, IED. }\end{array}$ & $\begin{array}{l}\text { Cointegración } \\
\text { para datos de } \\
\text { panel (vecM), } \\
\text { causalidad de } \\
\text { Granger. }\end{array}$ & $\mathrm{Y} \rightarrow \mathrm{CO}_{2}$ \\
\hline $\begin{array}{l}\text { Balado- } \\
\text { Naves et al. } \\
(2018)\end{array}$ & $1990-2014$ & $\begin{array}{l}173 \text { países } \\
\text { (incluido ALC) }\end{array}$ & $\begin{array}{l}\text { Emisiones de } \mathrm{CO}_{2 \prime} \\
\text { PIB, consumo de } \\
\text { energía, valor } \\
\text { agregado de ser- } \\
\text { vicios, intensidad } \\
\text { energética. }\end{array}$ & $\begin{array}{l}\text { Mco dinámi- } \\
\text { co, modelo de } \\
\text { error espacial de } \\
\text { Durbin. }\end{array}$ & $\begin{array}{l}\text { EKC } \\
(2)\end{array}$ \\
\hline $\begin{array}{l}\text { Wang et al. } \\
(2017)\end{array}$ & $1980-2011$ & $\begin{array}{l}170 \text { países } \\
\text { (incluido ALC) } \\
\text { agrupador } \\
\text { por ingreso }\end{array}$ & $\begin{array}{l}\text { Urbanización, } \\
\text { PIB, consumo } \\
\text { de energía, } \\
\text { emisiones de } \mathrm{CO}_{2} \text {. }\end{array}$ & $\begin{array}{l}\text { Causalidad de } \\
\text { Granger, } \\
\text { funciones } \\
\text { de impulso- } \\
\text { respuesta. }\end{array}$ & $\mathrm{CO}_{2} \leftrightarrow \mathrm{Y}$ \\
\hline $\begin{array}{l}\text { Santana de } \\
\text { Souza et al. } \\
(2018)\end{array}$ & $1990-2014$ & Mercosur & $\begin{array}{l}\text { Emisiones de } \\
\mathrm{CO}_{2} \text {, consumo de } \\
\text { energía, PIB }\end{array}$ & Panel мco. & $\mathrm{Y}+\mathrm{CO}_{2}$ \\
\hline $\begin{array}{l}\text { Zambrano- } \\
\text { Monserrate } \\
\text { et al. (2018) }\end{array}$ & $1980-2011$ & $\begin{array}{l}\text { Países de } \\
\text { América } \\
\text { Latina }\end{array}$ & $\begin{array}{l}\text { Emisiones de } \mathrm{CO}_{2} \text { ' } \\
\text { PIB, consumo de } \\
\text { energía, consumo } \\
\text { de gas natural } \\
\text { pc, consumo de } \\
\text { petróleo pc. }\end{array}$ & $\begin{array}{l}\text { ARDL. Causali- } \\
\text { dad de Granger, } \\
\text { cointegración. } \\
\text { vECM. }\end{array}$ & $\mathrm{CO}_{2} \rightarrow \mathrm{Y}$ \\
\hline $\begin{array}{l}\text { Acheam- } \\
\text { pong } \\
(2018)\end{array}$ & $1990-2014$ & $\begin{array}{l}116 \text { países } \\
\text { (incluido ALC) }\end{array}$ & $\begin{array}{l}\text { Consumo de ener- } \\
\text { gía, } \mathrm{PIB} \text {, emisiones } \\
\text { de } \mathrm{CO}_{2} \text {, apertura } \\
\text { comercial. }\end{array}$ & $\begin{array}{l}\text { PVAR } \\
\text { MGM }\end{array}$ & $\begin{array}{l}\mathrm{Y} \leftrightarrow \mathrm{CO}_{2} \\
\mathrm{CO}_{2} \rightarrow \mathrm{Y}(\mathrm{AL})\end{array}$ \\
\hline
\end{tabular}




\begin{tabular}{|c|c|c|c|c|c|}
\hline Trabajo & $\begin{array}{l}\text { Periodo } \\
\text { analizado }\end{array}$ & Países & Variables & Metodología & Conclusiones \\
\hline $\begin{array}{l}\text { De Souza } \\
\text { et al. } \\
\text { (2018) }\end{array}$ & 1990-2014 & Mercosur & $\begin{array}{l}\text { Emisiones de } \\
\mathrm{CO}_{2} \text {, consumo de } \\
\text { energía, PIB. }\end{array}$ & Efectos fijos. & $\begin{array}{l}\mathrm{EKC} \\
\mathrm{CER}-\mathrm{CO}_{2} \\
\mathrm{CENR}+\mathrm{CO}_{2}\end{array}$ \\
\hline $\begin{array}{l}\text { Saidi y } \\
\text { Hammami } \\
\text { (2017) }\end{array}$ & 2000-2014 & $\begin{array}{l}75 \text { países } \\
\text { (incluido ALC) }\end{array}$ & $\begin{array}{l}\text { Consumo de ener- } \\
\text { gía, desarrollo fi- } \\
\text { nanciero, emisio- } \\
\text { nes de } \mathrm{Co}_{2} \text {, stock } \\
\text { de capital, aper- } \\
\text { tura comercial, } \\
\text { urbanización. }\end{array}$ & $\begin{array}{l}\text { Panel dinámico } \\
\text { por GMM. }\end{array}$ & $\mathrm{CO}_{2}+\mathrm{Y}$ \\
\hline $\begin{array}{l}\text { Rodríguez- } \\
\text { Caballero } \\
\text { y Ventosa- } \\
\text { Santaularia } \\
\text { (2017) }\end{array}$ & $\begin{array}{l}\text { Últimos } \\
30 \text { años }\end{array}$ & $\begin{array}{l}17 \text { países de } \\
\text { ALC, Canadá y } \\
\text { EE. UU. }\end{array}$ & $\begin{array}{l}\text { Consumo } \\
\text { primario de } \\
\text { energía, PIB. }\end{array}$ & $\begin{array}{l}\text { Causalidad de } \\
\text { Granger, } \\
\text { especificación } \\
\text { VEC. }\end{array}$ & $\begin{array}{l}\text { CEE } \rightarrow \text { Y }(8 \\
\text { países }) \\
\text { Y } \rightarrow \text { CEE (3 } \\
\text { países) }\end{array}$ \\
\hline $\begin{array}{l}\text { Ozturk } \\
(2017)\end{array}$ & $1975-2013$ & $\begin{array}{l}\text { América La- } \\
\text { tina }\end{array}$ & $\begin{array}{l}\text { Consumo de } \\
\text { energía, emisio- } \\
\text { nes de } \mathrm{CO}_{2} \text {, IED, PIB } \\
\text { pc, retornos del } \\
\text { petróleo. }\end{array}$ & $\begin{array}{l}\text { Método del } \\
\text { Zellner, } \\
\text { causalidad de } \\
\text { Granger. }\end{array}$ & $\mathrm{Y} \rightarrow \mathrm{CO}_{2}$ \\
\hline $\begin{array}{l}\text { Antonaka- } \\
\text { kis et al. } \\
\text { (2017) }\end{array}$ & 1971-2011 & $\begin{array}{l}106 \text { países } \\
\text { (incluido ALC) } \\
\text { agrupados } \\
\text { por ingreso }\end{array}$ & $\begin{array}{l}\mathrm{PIB} \text {, emisiones de } \\
\mathrm{CO}_{2} \text {, consumo de } \\
\text { energía. }\end{array}$ & $\begin{array}{l}\text { PVAR. Funciones } \\
\text { impulso- } \\
\text { respuesta. }\end{array}$ & $\mathrm{Y} \leftrightarrow \mathrm{CE}$ \\
\hline Ito (2017) & 2002-2011 & $\begin{array}{l}42 \text { países en } \\
\text { desarrollo } \\
\text { (incluido ALC) }\end{array}$ & $\begin{array}{l}\text { Emisiones de } \mathrm{CO}_{2} \\
\text { pc, consumo de } \\
\text { energía, PIB. }\end{array}$ & $\begin{array}{l}\text { Panel oLs. GMM } \\
\text { Arellano-Bond. } \\
\text { ARDL. }\end{array}$ & $\begin{array}{l}\text { CER }+Y \\
\text { CENR-Y }\end{array}$ \\
\hline $\begin{array}{l}\text { Dutta y Das } \\
\text { (2016) }\end{array}$ & $1960-2010$ & $\begin{array}{l}60 \text { países } \\
\text { (incluido } 6 \\
\text { países de ALC) }\end{array}$ & $\begin{array}{l}\mathrm{PIB}, \text { emisiones de } \\
\mathrm{CO}_{2} .\end{array}$ & $\begin{array}{l}\text { Panel vaR, } \\
\text { causalidad de } \\
\text { Granger. }\end{array}$ & $\mathrm{Y} \rightarrow \mathrm{CO}_{2}$ \\
\hline $\begin{array}{l}\text { Narayan } \\
\text { et al. (2015) }\end{array}$ & $1960-2008$ & $\begin{array}{l}181 \text { países } \\
\text { (incluido ALC) }\end{array}$ & $\begin{array}{l}\mathrm{PIB}, \text { emisiones de } \\
\mathrm{CO}_{2} .\end{array}$ & $\begin{array}{l}\text { Coeficiente de } \\
\text { correlación } \\
\text { cruzada. }\end{array}$ & $\begin{array}{l}12 \% \text { EKC } \\
27 \% \text { reduc- } \\
\text { ción } \mathrm{CO}_{2} \text { a } \\
\text { mayor ingre- } \\
\text { so a LP }\end{array}$ \\
\hline $\begin{array}{l}\text { Apergis y } \\
\text { Payne } \\
\text { (2015) }\end{array}$ & $1980-2010$ & $\begin{array}{l}11 \text { países de } \\
\text { América del } \\
\text { Sur }\end{array}$ & $\begin{array}{l}\text { PІв pc, emisiones } \\
\text { de } \mathrm{CO}_{2} \text { pc, precio } \\
\text { del petróleo, con- } \\
\text { sumo de energía. }\end{array}$ & $\begin{array}{l}\text { Causalidad de } \\
\text { Granger. }\end{array}$ & $\mathrm{Y} \leftrightarrow \mathrm{CO}_{2}$ \\
\hline $\begin{array}{l}\text { Al Mulali } \\
\text { (2014) }\end{array}$ & $1990-2010$ & $\begin{array}{l}30 \text { países } \\
\text { consumidores } \\
\text { de energía } \\
\text { nuclear }\end{array}$ & $\begin{array}{l}\text { Emisiones de } \\
\mathrm{CO}_{2} \text {, PIB, consumo } \\
\text { de electricidad, } \\
\text { inversión domés- } \\
\text { tica, cantidad de } \\
\text { trabajadores. }\end{array}$ & $\begin{array}{l}\text { Causalidad de } \\
\text { Granger. }\end{array}$ & $\mathrm{Y} \rightarrow \mathrm{CO}_{2}$ \\
\hline
\end{tabular}




\begin{tabular}{|c|c|c|c|c|c|}
\hline Trabajo & $\begin{array}{c}\text { Periodo } \\
\text { analizado }\end{array}$ & Países & Variables & Metodología & Conclusiones \\
\hline $\begin{array}{l}\text { Al Mamun } \\
\text { et al. } \\
(2014)\end{array}$ & 1980-2009 & $\begin{array}{l}136 \text { países } \\
\text { (incluido ALC) }\end{array}$ & $\begin{array}{l}\text { PIB, apertura } \\
\text { comercial, valor } \\
\text { agregado (agro., } \\
\text { ind., serv.), emi- } \\
\text { siones de } \mathrm{CO}_{2} \text {. }\end{array}$ & $\begin{array}{l}\text { Cointegración: } \\
\text { método de } \\
\text { Westerlund. }\end{array}$ & $\begin{array}{l}\text { EKC (salvo en } \\
\text { países de la } \\
\text { OCDE de altos } \\
\text { ingresos) }\end{array}$ \\
\hline $\begin{array}{l}\text { Apergis y } \\
\text { Payne } \\
(2014)\end{array}$ & $1980-2010$ & $\begin{array}{l}7 \text { países de } \\
\text { América } \\
\text { Central }\end{array}$ & $\begin{array}{l}\text { PIB, consumo } \\
\text { de electricidad, } \\
\text { emisiones de } \mathrm{CO}_{2} \text {, } \\
\text { precio del petró- } \\
\text { leo y del carbón. }\end{array}$ & $\begin{array}{l}\text { Cointegración } \\
\text { no lineal, } \\
\text { causalidad de } \\
\text { Granger. }\end{array}$ & $\mathrm{Y} \leftrightarrow \mathrm{CO}_{2}$ \\
\hline $\begin{array}{l}\text { Al Mulali } \\
\text { et al. } \\
(2013)\end{array}$ & $1980-2008$ & $\begin{array}{l}32 \text { países de } \\
\text { ALC }\end{array}$ & $\begin{array}{l}\text { PIB, consumo pri- } \\
\text { mario de energía, } \\
\text { emisiones de } \mathrm{CO}_{2} \text {. }\end{array}$ & $\begin{array}{l}\text { Estimación } \\
\text { canónica } \\
\text { integrada. }\end{array}$ & $\mathrm{y} \leftrightarrow \mathrm{CO}_{2}$ \\
\hline $\begin{array}{l}\text { Apergis y } \\
\text { Payne (2009) }\end{array}$ & $1971-2004$ & $\begin{array}{l}6 \text { países } \\
\text { América } \\
\text { Central }\end{array}$ & $\begin{array}{l}\text { PIB, consumo de } \\
\text { energía, emisio- } \\
\text { nes de } \mathrm{CO}_{2} \text {. }\end{array}$ & vecm & $\begin{array}{l}\mathrm{y} \leftrightarrow \mathrm{CO}_{2} \\
\mathrm{y} \rightarrow \mathrm{CO}_{2}\end{array}$ \\
\hline
\end{tabular}

Y: crecimiento económico.

$C E$ : consumo de energía.

$C E E$ : consumo de energía eléctrica.

CER: consumo de energía renovable.

CENR: consumo de energía no renovable.

VECM: vector error correction model.

$I E D$ : inversión extranjera directa.

ARDL: modelos autorregresivos de retardos distribuidos.

UECM: modelos irrestrictos de corrección del error.

IAA: innovation accounting aproach.

PVAR: vectores autorregresivos para paneles.

MGM: método generalizado de los momentos.

$A \rightarrow \mathrm{B}$ : relación causal de A hacia $\mathrm{B}$.

$A+(-)$ B: impacto positivo (negativo) de A en B (sin testeo de causalidad).

(1) Para altos niveles de institucionalidad, el incremento del producto implica aumentos más suavizados de emisiones de dióxido de carbono.

(2) Con predicciones hasta 2100 se obtiene que el crecimiento acelerará el cambio climático.

Fuente: elaboración propia.

\section{Datos, metodología y resultados}

En esta sección se presentan los datos empleados, la metodología utilizada y resultados obtenidos. En primer lugar, se realiza la simbolización de las series temporales bidimensionales, lo que permite introducir una noción de distancia entre los países para luego realizar el análisis de cluster, y así poder agrupar a los países de la muestra en función del grado de similitud en su desempeño dinámico. Por último, para cada uno de los grupos obtenidos se aplican técnicas econométricas para estudiar la relación existente entre las 
variables, se testea causalidad, cointegración y se realizan las estimaciones de los modelos econométricos, aplicando distintos métodos para mayor robustez.

\subsection{Datos}

El crecimiento económico es representado mediante la tasa de crecimiento del Рів per cápita (en porcentajes), con datos obtenidos del Proyecto Maddison (2018) a partir del indicador PIB real comparable entre países. Las mediciones de emisiones de $\mathrm{CO}_{2}$ se obtuvieron del trabajo de Boden, Anders y Marland (2017). En particular, se consideran las emisiones de $\mathrm{CO}_{2}$ medidas en términos per cápita (en miles de toneladas métricas) a fin de recoger el tamaño de las economías y obtener un resultado más ajustado en términos interpretativos. La medición de emisiones de $\mathrm{CO}_{2}$ incluyen el consumo de combustible en cualquiera de sus estados: gas, líquido y sólido, medidos en toneladas métricas por habitante. ${ }^{1}$ En función de la disponibilidad de datos de ambas fuentes y con el fin de trabajar con series completas y la mayor cantidad de países, la muestra está compuesta por 24 países de América Latina y el Caribe: Argentina, Barbados, Bolivia, Brasil, Chile, Colombia, Costa Rica, Cuba, Dominica, Ecuador, El Salvador, Guatemala, Haití, Honduras, Jamaica, México, Nicaragua, Panamá, Paraguay, Perú, República Dominicana, Santa Lucía, Uruguay y Venezuela, considerando un periodo de 64 años comprendido entre 1951-2014. ${ }^{2}$

\subsection{Simbolización de series}

Daw et al. (2003) introduce el análisis de series temporales simbólicas, que junto al concepto de "régimen económico" mencionado en Brida et al. (2013) permite simbolizar series temporales multidimensionales e introducir una métrica para comparar el desempeño dinámico de los países, y a partir de esto realizar un análisis de cluster. En particular, la simbolización de series temporales ha sido empleada ampliamente en estudios sobre sistemas dinámicos, y para capturar las propiedades cualitativamente relevantes se introduce la dinámica de regímenes. La transformación de las series temporales bidimensionales en series simbólicas se justifica por diferentes motivos.

1 Es relevante destacar que el indicador considerado no incluye las emisiones de metano, elemento importante para las economías ganaderas, entre otros.

2 El periodo de análisis comienza en 1951, pues a partir de este año se tienen datos de la tasa del crecimiento del PIв y finaliza en el 2014, último año para el cual se tienen mediciones de emisiones de $\mathrm{CO}_{2}$. 
Entonces bien, al trabajar con series temporales de dimensión mayor a uno, en general, no se sabe si existe alguna relación entre las unidades de medida de las variables que intervienen y muchas veces esta relación no existe. Lo anterior impide el uso de métricas euclidianas o similares, visto que estas parten del supuesto que las unidades de medida en los ejes coordenados son las mismas. La metodología de simbolización de las series permite superar este problema. Además, para analizar la dinámica de forma cualitativa se introduce el concepto de régimen económico, que de acuerdo con la dinámica de regímenes admite una representación mediante series simbólicas. ${ }^{3}$

Intuitivamente, se puede interpretar a un régimen económico como un comportamiento particular en términos cualitativos que se diferencia de otros. Para este caso, cada régimen corresponde a una performance económica particular, diferenciada de los otros regímenes. En este caso, la partición elegida está determinada por los promedios anuales de la tasa de crecimiento del PIB per cápita $\left(\mu_{x t}\right)$ y de las emisiones de $\mathrm{CO}_{2}$ per cápita $\left(\mu_{y t}\right)$. Se debe tener en cuenta que la elección de estas medidas utilizadas para la determinación de los regímenes es exógena, y por ende, los resultados dependen de estas. Para cada año $t$ (con $t=1, \ldots, 64)$ se definen las medias de la tasa de crecimiento del PIB per cápita y de las emisiones de $\mathrm{CO}_{2}$ per cápita: $\mu_{x t}=\frac{1}{24} \sum_{i=1}^{24} x_{i t}$ y $\mu_{y t}=\frac{1}{24} \sum_{i=1}^{24} y_{i t}$.

Así, a partir de la partición elegida se obtienen cuatro regímenes según el cuadrante:

$$
\begin{aligned}
& R_{1}=\left\{\left(x_{t}, y_{t}\right): x_{t} \leq \mu_{x_{t}}, y_{t} \leq \mu_{y_{t}}\right\} \\
& R_{2}=\left\{\left(x_{t}, y_{t}\right): x_{t} \geq \mu_{x_{t}}, y_{t} \leq \mu_{y_{t}}\right\} \\
& R_{3}=\left\{\left(x_{t}, y_{t}\right): x_{t} \geq \mu_{x_{t}}, y_{t} \geq \mu_{y_{t}}\right\} \\
& R_{2}=\left\{\left(x_{t}, y_{t}\right): x_{t} \leq \mu_{x_{t}}, y_{t} \geq \mu_{y_{t}}\right\}
\end{aligned}
$$

Al aplicar el criterio de partición definido con anterioridad, la figura 1 muestra la dispersión en el espacio de los puntos correspondientes al nivel de tasa de crecimiento del producto real per cápita y las emisiones de $\mathrm{CO}_{2}$ per cápita para el 2014, a modo de ejemplo para cada uno de los 24 países.

3 Al lector interesado en el análisis de series temporales simbólicas y sus aplicaciones a la economía se le sugiere consultar el reciente trabajo de Risso (2019). 


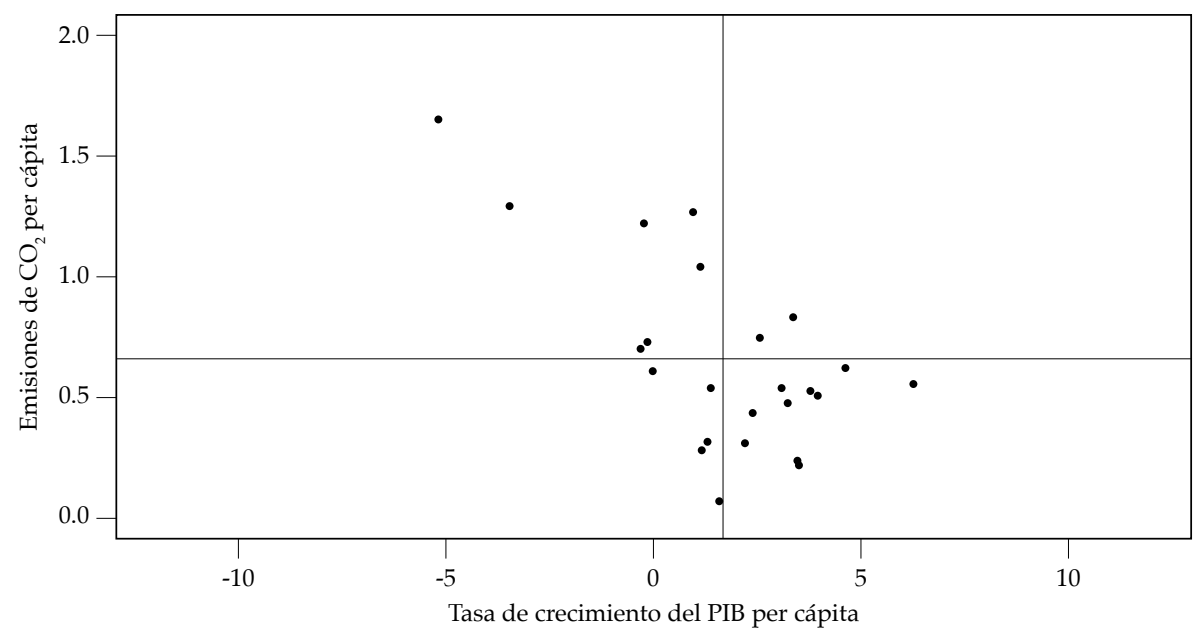

Figura 1. Nube de puntos para los 24 países en el 2014

Fuente: elaboración propia.

Contando desde la partición del espacio en regímenes, se distinguen dos tipos de dinámicas, una dentro de cada régimen y otra de cambio entre regímenes. La dinámica observada en cada régimen unido al conjunto de la partición elegida es lo que determina un modelo de desempeño que se diferencia de los modelos de los otros conjuntos de la partición. La dinámica de cambio de regímenes puede ser representada de la siguiente manera: a cada país se le asigna un símbolo (1, 2, 3 o 4) en cada momento del tiempo, dependiendo de la región en que esté ubicado. ${ }^{4}$ Se le asigna un símbolo a cada región (en este caso, la etiqueta elegida es el número de régimen). Así, se transforma la serie temporal bidimensional de crecimiento económico y emisiones de dióxido de carbono $\left(x_{t}, y_{t}\right)$ donde $t$ toma los valores enteros comprendidos entre 1951 y el 2014, en la serie temporal simbólica $s=\left\{\left(x_{1}, y_{1}\right)\right.$, $\left.\left(x_{2}, y_{2}\right), \ldots,\left(x_{T}, y_{T}\right)\right\}$ de modo tal que $s_{t}=j$ si y solo si $\left(x_{t}, y_{t}\right)$ está en el régimen $R_{j}$. La serie simbólica $s=\left\{\left(x_{1}, y_{1}\right),\left(x_{2}, y_{2}\right), \ldots,\left(x_{T}, y_{T}\right)\right\}$ contiene toda la información relevante acerca de la dinámica de regímenes poniendo en evidencia distintos tipos de desempeño de los países.

Se pueden observar países donde la permanencia estuvo dada casi siempre en regímenes con bajos niveles de emisiones per cápita, y en oposición, otros casos donde la permanencia estuvo dada en niveles de altas emisiones. Sin embargo, ciertos casos muestran que la permanencia es alternada entre

4 Se utiliza $\geq \mathrm{y} \leq$ en la frontera de los regímenes, pues la probabilidad de que un país pertenezca a dos regiones en un mismo periodo es nula. 
los cuatro distintos regímenes como comportamientos dinámicos disímiles a lo largo del periodo, pero donde existe cierta predominancia en la permanencia en un régimen determinado (véase tabla A1 en el Apéndice).

La figura 2 muestra el comportamiento de algunos países, incorporando la dimensión temporal. Como se puede observar, países como Brasil y Costa Rica en la gran mayoría del periodo permanecen entre el régimen 1 y 2 , es decir, fluctuando en términos de su tasa de crecimiento y con nivel de emisiones de dióxido de carbono bajas (por debajo del promedio del panel). Se debe tener en cuenta que, las emisiones se consideran en términos de la población, por lo que si bien Brasil presenta bajas emisiones per cápita, esto se debe en gran parte al tamaño de la población, ya que en términos agregados las emisiones totales son muy altas, mientras que Costa Rica presenta niveles bajos ya sea que se considere en términos agregados o per cápita. Por otro lado, se observan países como Argentina y Barbados, con un nivel de emisiones constantemente altos $y$, tasas de crecimiento que varían por encima y por debajo del promedio.

Evolución de Brasil

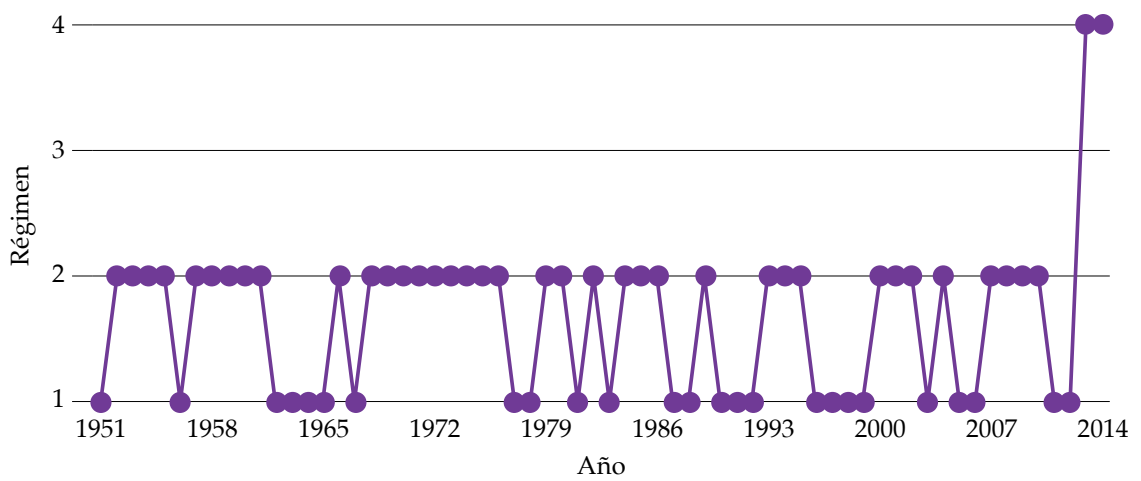

Evolución de Argentina

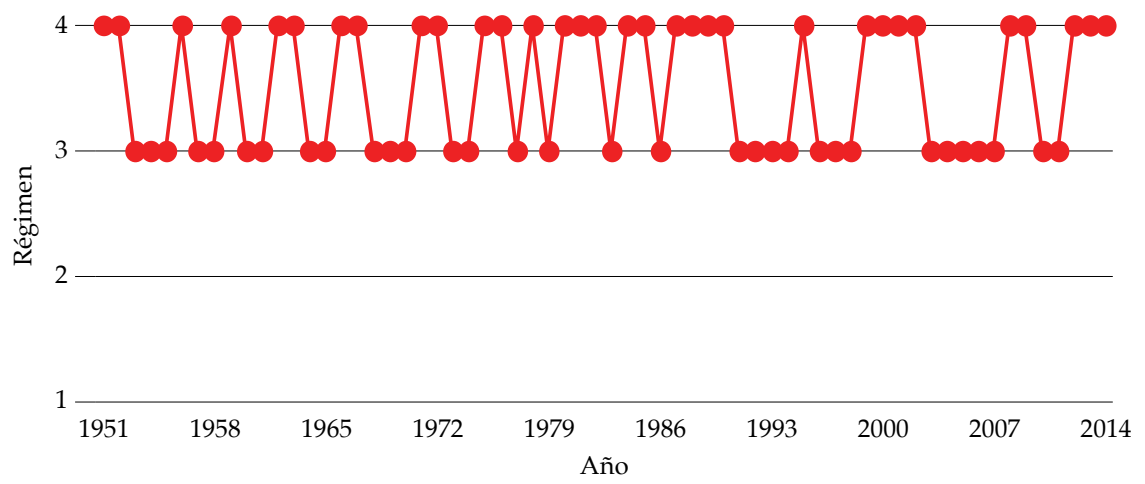




\section{Evolución de Costa Rica}

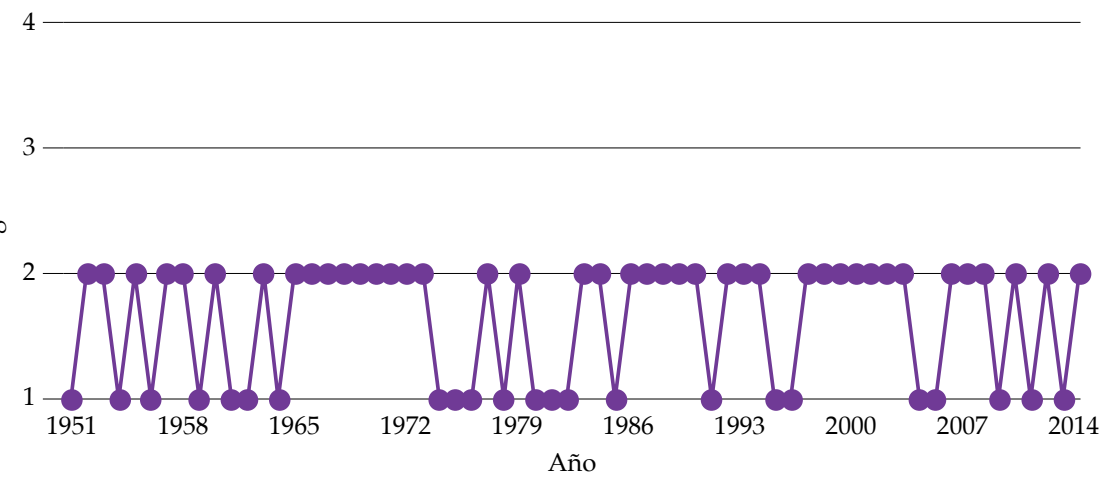

Evolución de Barbados

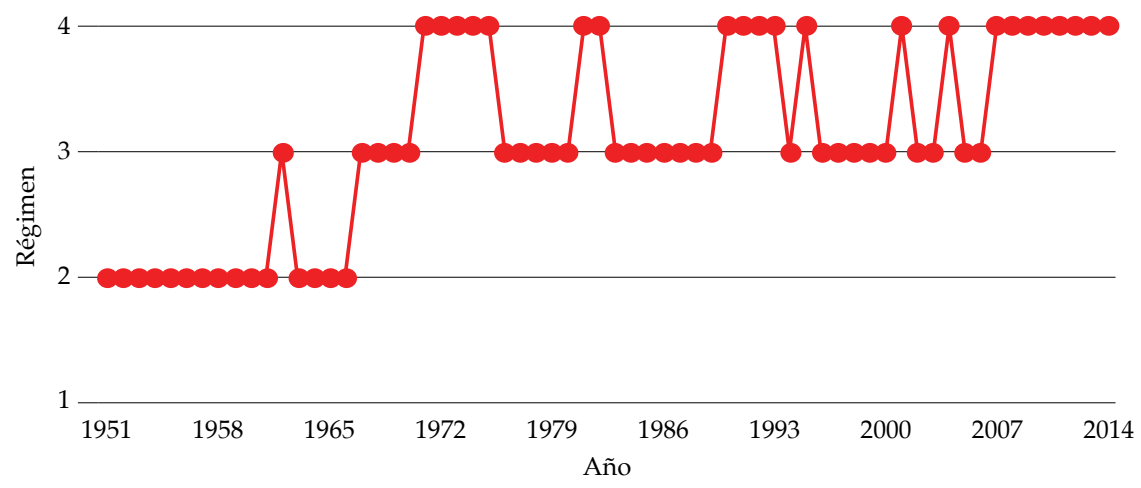

Evolución de Uruguay

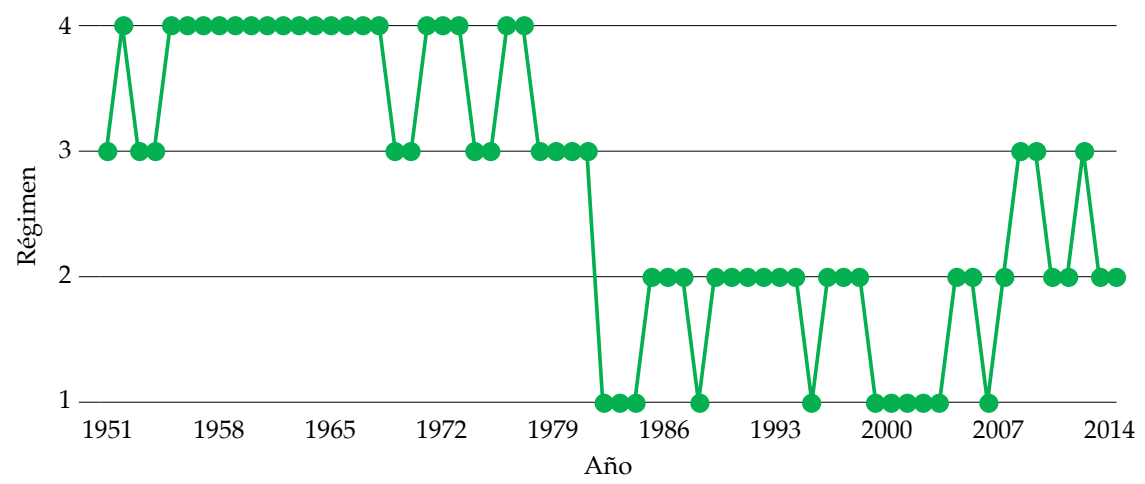




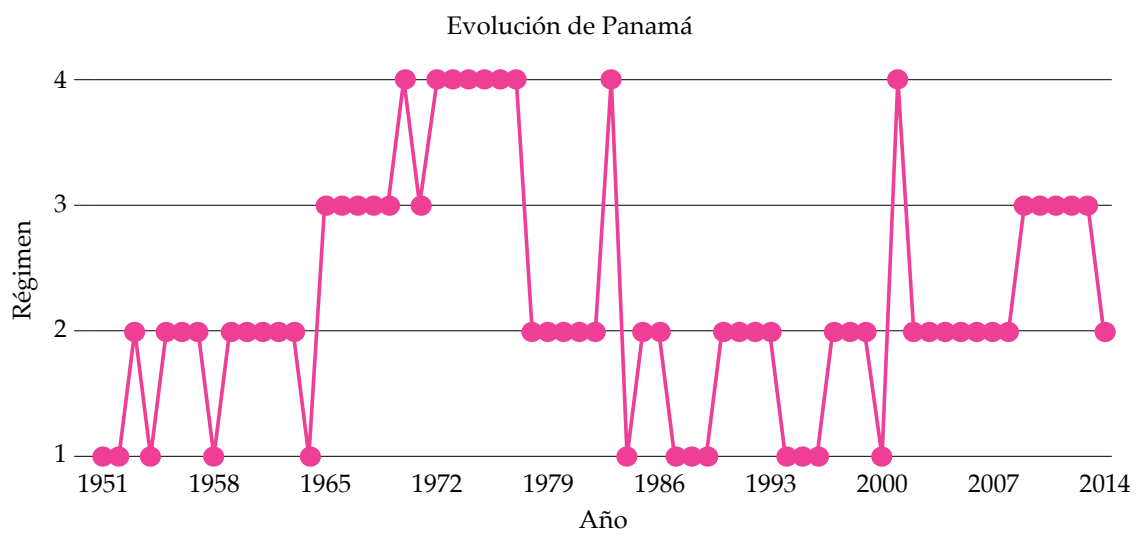

Figura 2. Trayectorias simbólicas de algunos países (1951-2014)

Fuente: elaboración propia.

En este caso también se ha de destacar la incidencia que tiene el tamaño de la población, pues en términos agregados, Argentina tiene emisiones considerablemente mayores que Barbados. Es interesante el comportamiento que exhibe Barbados, ubicándose al inicio del periodo y durante 15 años aproximadamente en el régimen 2, y luego pasa a ubicarse en los regímenes 3 y 4 durante lo que resta del periodo, es decir, que inicia con bajas emisiones (y alta tasa de crecimiento económico) y hacia el final de los años 60 comienza a posicionarse en los regímenes de alta emisión de $\mathrm{CO}_{2}$. Además, es interesante el comportamiento que tienen Uruguay y Panamá, que no presentan el mismo comportamiento de los otros países. Se puede observar que ambos recorren todos los regímenes y por tanto a lo largo del periodo han tenido grandes fluctuaciones en cuanto a emisiones y crecimiento. Una primera aproximación interpretativa podría ser la idea de que ambos países — aún con dinámicas poco estables entre regímenes-, durante el periodo parecen permanecer mayor tiempo en un contexto de bajas emisiones de dióxido de carbono.

\subsection{Análisis de cluster}

Una vez realizada la simbolización — habiendo obtenido las series simbólicas que dan prueba de las trayectorias dinámicas cualitativas de los países en términos de emisiones de $\mathrm{CO}_{2}$ y crecimiento económico-, es posible introducir una noción de distancia (véase Tang et al., 1997; Molgedey \& Ebeling, 2000; Piccardi, 2004) que permita analizar la similitud en el comportamiento de los países y estudiar la existencia de grupos de países con dinámicas semejantes. La distancia entre dos países $i$ y $j$ se define como: 


$$
\begin{aligned}
& d\left(s_{i}, s_{j}\right)=\sum_{t=1}^{64} f\left(s_{i t}, s_{j t}\right), \text { donde } f\left(s_{i}, s_{j}\right)=\left\{\begin{array}{l}
0 \text { si } s_{i t}=s_{j t} \\
1 \text { si } s_{i t} \neq s_{j t}
\end{array} \text { con } t=1, \ldots, 64 ;\right. \\
& i=1, \ldots, 24
\end{aligned}
$$

Con la información derivada de la matriz de distancias obtenida para los 24 países considerados, se puede construir el Dendograma o Árbol Jerárquico (HT por sus siglas en inglés, Hierarchical Tree) donde se aplica la metodología del "Vecino más cercano" (Mantegna, 1999; Mantegna \& Stanley, 2000). En la figura 3 se presenta el HT, en el que se puede observar claramente la conformación de dos grupos con características similares y dos países que no se integran a ninguno de estos grupos (a partir de la especificación sucesiva de modelos jerárquicos y del criterio de información de Schwarz — o criterio de información bayesiano- se obtiene que el número óptimo de grupos es 4).

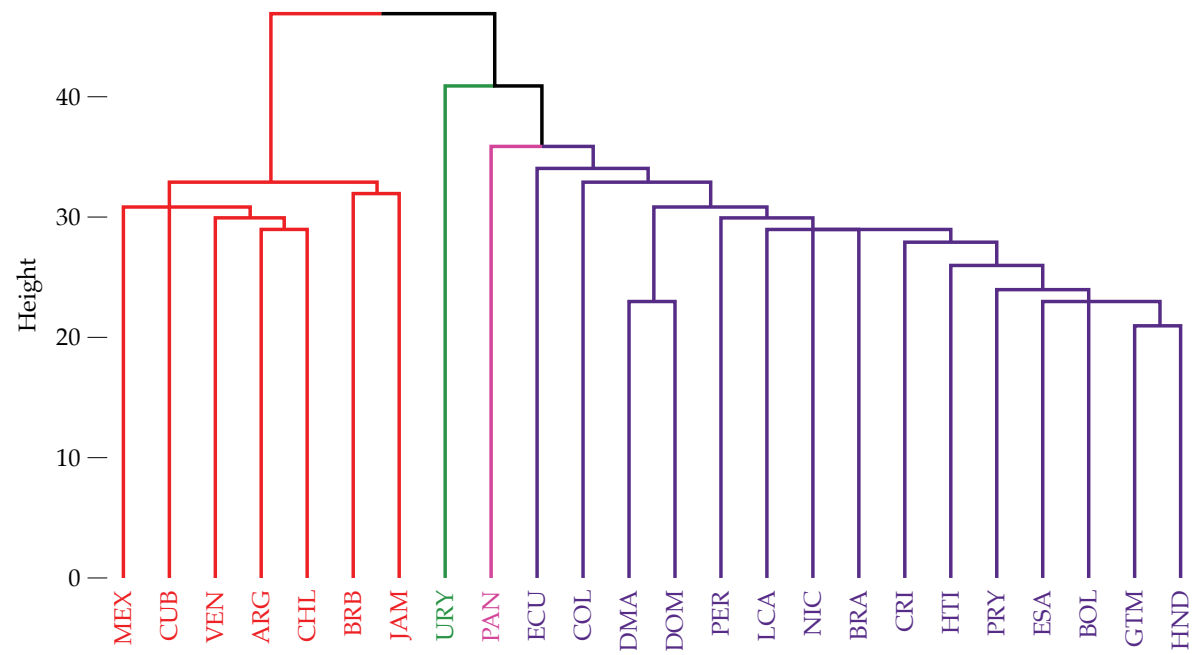

Figura 3. Árbol Jerárquico (1951-2014)

Fuente: elaboración propia en R.

De acuerdo con la figura 3 se puede visualizar la conformación de los grupos, interpretados de la siguiente manera:

1. Países con un nivel de emisiones de $\mathrm{CO}_{2}$ elevado en términos per cápita y con un ambiente más degradado. En cuanto a régimen económico son países que han transitado entre el régimen 3 y 4: Jamaica, Barbados, Chile, Argentina, México, Venezuela y Cuba. 
2. Países con niveles reducidos de emisiones de $\mathrm{CO}_{2}$ per cápita y por tanto con un mejor rendimiento en materia ambiental, alternando su dinámica entre los regímenes 1 y 2: Perú, Ecuador, Dominica, República Dominicana, Santa Lucía, Nicaragua, Brasil, Colombia, Haití, Costa Rica, Paraguay, El Salvador, Guatemala, Honduras y Bolivia.

3. Uruguay, un caso particular con una dinámica muy dispar en el tiempo, pese a que ha tenido un periodo de altas emisiones (regímenes 3 y 4), parece haber encontrado un rumbo en el último tiempo con bajas emisiones, aunque con un crecimiento fluctuante.

4. Panamá, otro caso particular que a lo largo de los años no logró una dinámica estable, sino que ha tenido altos y bajos en materia ambiental.

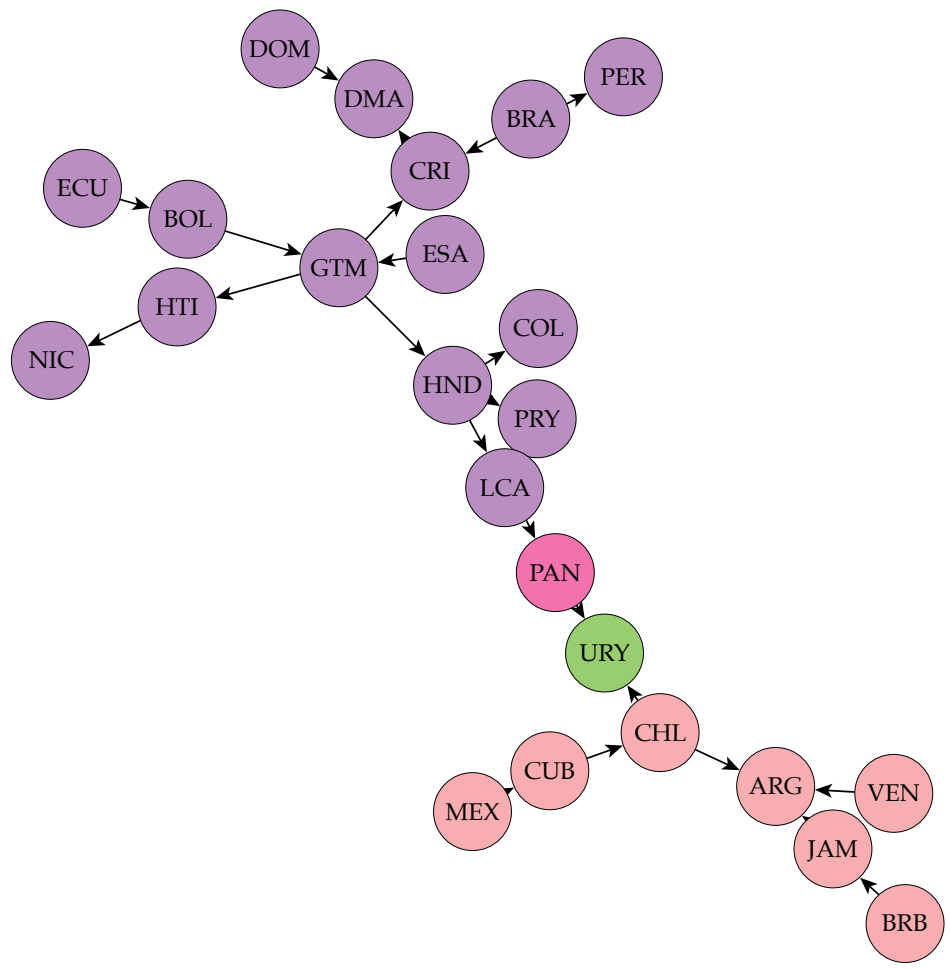

Figura 4. Árbol de Expansión Mínima (1951-2014)

Fuente: elaboración propia en $\mathrm{R}$.

En la figura 4 se observa el Árbol de Expansión Mínima (MST por sus siglas en inglés, Minimum Spanning Tree), el cual se construye a partir del algoritmo de Kruskal (1956). Partiendo del MST se pueden analizar algunos rasgos característicos que influyen sobre el comportamiento de los países. 
En primer lugar, es posible observar los dos clusters de forma clara, los cuales se encuentran conectados de acuerdo a los países que se consideran outliers; aquellos que no mantienen una dinámica común a ninguno de los dos grupos. Panamá parece tener una dinámica más próxima al grupo de bajas emisiones convergiendo con ese grupo a una menor distancia de lo que lo hace Uruguay. Sin embargo, Uruguay también, aunque a una distancia mayor se ajusta de mejor forma (en términos de distancia) al grupo de reducidas emisiones en comparación al grupo de emisiones elevadas. En segundo lugar, se puede observar que Chile y Santa Lucía son los dos países de cada grupo más expuestos al cambio de grupo, y por tanto, con una dinámica más próxima a otro comportamiento distinto de la del grupo en su conjunto. No obstante, siguiendo el índice propuesto por Krackhardt y Stern (1988), ${ }^{5}$ hay evidencia a favor de que estos países tienen un comportamiento más similar al grupo al que efectivamente pertenece, y se aleja de la idea de un potencial cambio de grupo. Considerando la aproximación de centralidad de grado, Guatemala es el país más central del panel (y por tanto de su grupo), mientras que Argentina es el país más central del grupo de altas emisiones. Esto puede aportar en el sentido de considerar sus dinámicas como potenciales influyentes en la dinámica del grupo en su conjunto.

A partir de los resultados obtenidos del análisis de cluster se pueden caracterizar dos grupos (véase figura A1 en el Apéndice). Uno de bajas emisiones de $\mathrm{CO}_{2}$ per cápita donde se agrupan 15 países, dentro de los cuales se encuentran algunas islas del caribe que no se caracterizan por el desarrollo de actividades contaminantes y que, por tanto, no emiten grandes cantidades de dióxido de carbono. Además, está integrado por algunos países que, si bien su nivel de emisiones medido en términos per cápita es reducido, cuando se observan sus niveles absolutos, son elevados. Este grupo emite en promedio 0.25 toneladas métricas de dióxido de carbono por habitante. En la dinámica se observa una trayectoria creciente de las emisiones por habitante asociada a una degradación ambiental cada vez mayor, vinculada principalmente a mayores actividades de explotación que tienen como consecuencia la emisión de estos gases. A su vez, esta trayectoria parece adecuarse a cierta tendencia sin sufrir demasiada variabilidad en el tiempo (véase tabla A2 y figura A2 en el Apéndice). Por otro lado, el segundo grupo de países es más pequeño, solo se conforma por 7 y se observan altos niveles de emisiones de $\mathrm{CO}_{2}$ per cápita. Estos países, además, tienen altos niveles absolutos de

5 Este indicador puede tomar valores entre - 1 y 1, donde -1 refleja la máxima homofilia y 1 máxima heterofilia. 
emisiones de $\mathrm{CO}_{2}$ debido a sus actividades, como por ejemplo, refinamiento de petróleo, servicios altamente contaminantes como el transporte, o países que tienen suficientes emisiones como para que, al medirlas en términos per cápita, alcancen un valor elevado en este indicador. Así, la serie promedio de emisiones alcanza su media en el periodo en 0.93 toneladas métricas por habitante. Esta serie se caracteriza por ser más volátil en el tiempo, en torno a una tendencia respecto a la descrita anteriormente, así mismo, de acuerdo a la serie promedio considerando a todo el panel de países, e incluso, su tendencia creciente es más acentuada que la del grupo 1 (véase tabla A2 y figura A2 en el Apéndice). Por último, en lo que respecta al crecimiento económico no se observa que un grupo se encuentre sistemáticamente con mejor rendimiento que otro, sin embargo, el crecimiento promedio para todo el periodo considerado es menor en el grupo 1. Los casos de Panamá y Uruguay se pueden considerar como outliers debido a que no se corresponden en su comportamiento dinámico conjunto con ninguno de los dos grupos antes descritos. Ambos países tienen un nivel de emisiones de $\mathrm{CO}_{2}$ per cápita promedio y un rango en sus niveles que no se corresponde a los esquemas plasmados en los dos grupos conformados por el resto de los países.

Para el estudio de la convergencia entre los grupos, se calcula el diámetro del MST (definido como la suma de enlaces entre los países), para cada grupo y para el total de los países, considerando ventanas temporales solapadas de 10 años cada una. Esto permite analizar la evolución del diámetro del MST, dando una aproximación a la potencial convergencia entre los países considerados (si el diámetro disminuye en el tiempo). Se observa que durante todo el periodo la trayectoria del diámetro de la muestra completa parece estable (a pesar de observarse una muy leve tendencia creciente). Sin embargo, considerando ambos grupos por separado, se observan distintos comportamientos. En cuanto al grupo de bajos niveles de emisiones, los países que lo conforman no parecen distanciarse. Por otra parte, observado el grupo de altos niveles de emisiones se encuentra que los países parecen estar más distanciados entre sí, en tendencia, dando lugar a dinámicas disímiles en el tiempo entre ellos. Adicionalmente la dinámica de evolución de las distancias parece tener una menor variabilidad si se la compara con la del primer grupo (véase figura A3 en el Apéndice).

\subsection{Una aproximación cuantitativa a la validación de la hipótesis de Kuznets para América Latina y el Caribe}

Habiendo obtenido grupos de países con similar desempeño, es posible aplicar técnicas cuantitativas que permitan analizar la validación empírica 
de la EKC para cada uno de los grupos. Para esto se aplicará una prueba de causalidad para paneles, a modo de evaluar la presencia de relaciones al menos en el corto plazo que verifiquen o refuten la relación teórica propuesta. Luego, considerando los resultados encontrados se realizará una aproximación preliminar a la cuantificación de los efectos encontrados. Para esta parte del análisis, se incluyen además las siguientes variables de control siguiendo a la literatura de referencia (véase Le \& Bach, 2020): gasto del gobierno per cápita (gasto), apertura comercial internacional (apertura) y formación bruta de capital fijo per cápita $(f b k f)$.

Para el estudio de las relaciones causales de largo plazo, muchos trabajos suelen utilizar pruebas de causalidad de Granger. Sin embargo, esta prueba considera un único modelo subyacente con una estructura paramétrica común a todos los países de la región. Dumitrescu y Hurlin (2012) proponen una alternativa que relaja la restricción anterior y posibilita la existencia de distintas estructuras paramétricas entre las unidades del panel, en este caso, los países. La metodología propuesta por Dumitrescu y Hurlin (2012) parte de la estimación de sucesivos modelos lineales para cada país, como se muestra en la ecuación (3) y a partir del test de significación individual sobre los coeficientes $\beta_{i k}$ y computando los estadísticos de Wald, se obtiene un estadístico de contraste con distribución asintóticamente normal estándar. ${ }^{6}$ La tabla 3 resume los resultados del estudio de las relaciones causales entre el nivel de actividad de las economías y sus emisiones de dióxido de carbono por habitante.

$$
y_{i t}=\gamma_{i 1} y_{i, t-1}+\cdots+\gamma_{i p} y_{i, t-p}+\beta_{i 1} x_{i, t-1}+\beta_{i p} x_{i, t-p}+\varepsilon_{i t}
$$

Tabla 3. Prueba de causalidad de Dumitrescu y Hurlin (2012)

\begin{tabular}{ccccc}
\hline Dumitrescu y Hurlin (2012) & \multicolumn{2}{c}{ Grupo 1 } & \multicolumn{2}{c}{ Grupo 2 } \\
\hline Hipótesis nula & Estad. & p-valor & Estad. & p-valor \\
\hline pibpc no causa emisiones & 8,5589 & 0,0000 & 5,0097 & 0,0000 \\
emisiones no causa pibpc & 12,0546 & 0,0000 & 4,8771 & 0,0000 \\
\hline
\end{tabular}

Fuente: elaboración propia.

Al aplicar este test a ambos grupos, se encuentra que para todo el periodo considerado existe una relación causal, al menos en el corto plazo en ambos sentidos entre el PIB real por habitante y las emisiones de $\mathrm{CO}_{2}$ per cápita (se

6 Para mayor detalle metodológico, véase López y Weber (2017). 
trabaja con las primeras diferencias, pues estas son estacionarias tal como requiere la prueba. En el apéndice se puede encontrar mayor información sobre las pruebas de raíz unitaria empleadas). A partir de estos resultados, sería relevante testear la existencia de relaciones de largo plazo entre estas variables y medir la magnitud de estas relaciones causales, en particular, para verificar la existencia de la EKC en América Latina y el Caribe.

A fin de evaluar la existencia de relaciones de largo plazo entre las series del producto de los países y sus emisiones de dióxido de carbono, se realiza el análisis de cointegración, empleando el test para paneles de Pedroni (1999, 2001). Con este se evalúa la existencia de potenciales relaciones lineales entre las variables que devuelven estacionariedad en las mismas, es decir que sean integradas de orden cero.

$$
c 02_{i t}=f_{i t}(\text { pib, gasto, apertura, } f b k f)+\mu_{i}+\varepsilon_{i t}
$$

Esta prueba parte de una hipótesis nula de no cointegración y una alternativa de que todos los paneles se encuentran cointegrados, partiendo de cierta especificación de un modelo y una determinación de sus rezagos. En este caso se consideran las variables previamente definidas y una cantidad óptima de rezagos determinada por AIC. Aplicando esta prueba, se encuentra que para ambos grupos existen relaciones de cointegración entre las variables dando evidencia de relaciones de largo plazo entre las series, siguiendo el estadístico $t$ de Phillip-Perron modificado se rechaza la hipótesis nula de no cointegración, al $5 \%$ de significación.?

Para la cuantificación de la relación existente entre las emisiones de $\mathrm{CO}_{2}$ y el nivel de actividad de los países, se formula el siguiente modelo lineal donde se incluye dentro del componente aleatorio un componente de heterogeneidad inobservable propio de cada uno de los países.

$$
c o 2_{i t}=\alpha+\beta_{1} p_{i b} b_{i t}+\beta_{2} \text { pib }_{i t}^{2}+\gamma_{1} \text { gasto }_{i t}+\gamma_{2} \text { apertura }_{i t}+\gamma_{3} f b k f_{i t}+\mu_{i}+\varepsilon_{i t}
$$

En la ecuación anterior, pib $_{i t}$ representa el logaritmo del PIB por habitante, $\mathrm{CO}_{i t}$ el logaritmo de las emisiones de $\mathrm{CO}_{2}$ per cápita, gasto ${ }_{i t}$ el logaritmo del gasto del Gobierno, apertura $_{i t}$ el logaritmo del índice de apertura de la economía, y $f b k f_{i t}$ la formación bruta de capital fijo para el país $i$, en el año $t$.

Para su estimación, en primer lugar, se emplea el método Mínimos Cuadrados Ordinarios bajo una especificación con Efectos Fijos (EF) y otra

7 Grupo 1: Estadístico $t$ de Phillip-Perron: -4,5617; p-valor: 0,0000. Grupo 2: Estadístico $t$ de Phillip-Perron: -1,4975; p-valor: 0,0671. 
con Efectos Aleatorios (EA) del componente de error. La tabla 4 muestra los resultados de las estimaciones puntuales de los coeficientes y los resultados de la aplicación del Test de Hausman. Este último sugiere cuál de las dos estimaciones es consistente y eficiente donde la hipótesis nula sugiere que el estimado por EF es consistente, pero EA es consistente y eficiente. Su hipótesis alternativa sugiere que EF es consistente, pero EA es inconsistente. A partir de los resultados obtenidos, se observa que en la mayoría de los casos los coeficientes estimados son significativos. Sin embargo, solo se logra verificar la EKC el grupo de bajas emisiones, ya sea que se estime mediante EF O EA. El testeo mediante Hausman da evidencia a favor de un estimador por EF consistente.

Tabla 4. Estimaciones por Efectos Fijos y Efectos Aleatorios. Test de Hausman

\begin{tabular}{|c|c|c|c|c|}
\hline & \multicolumn{2}{|c|}{ Grupo 1} & \multicolumn{2}{|c|}{ Grupo 2} \\
\hline & $\mathrm{EF}$ & EA & $\mathrm{EF}$ & EA \\
\hline cons & $-17,13481^{* * *}$ & $-14,69081^{* * *}$ & 4,587017 & $-0,2424542^{* * *}$ \\
\hline pib & $3,135057^{* * *}$ & $2,523889^{* * *}$ & $-1,444054$ & $-0,3559082$ \\
\hline$p i b^{2}$ & $-0,1645799^{* * *}$ & $-0,1179607^{* * *}$ & 0,0817519 & 0,0342026 \\
\hline gasto & $0,0981975^{* * *}$ & $-0,0270586$ & $0,2405895^{* * *}$ & 0,0644359 \\
\hline apertura & $0,2443834^{* * *}$ & $0,2441661^{* * *}$ & 0,041171 & 0,0523356 \\
\hline fbkf & $0,3898449^{* * *}$ & $0,2561351^{* * *}$ & $0,2217772^{* * *}$ & $0,1053385^{* * *}$ \\
\hline$R^{2}$ & 0,0315 & 0,1004 & 0,2222 & 0,3235 \\
\hline$F$ & $242,33^{* * *}$ & $1015,63^{* * *}$ & $47,93^{* * *}$ & $192,81^{* * *}$ \\
\hline Hausman & \multicolumn{2}{|c|}{$80,72^{* * *}$} & \multicolumn{2}{|c|}{$30,46^{* * *}$} \\
\hline
\end{tabular}

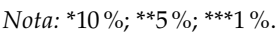

Fuente: elaboración propia.

A modo de dar robustez y contrastar estos resultados obtenidos, se propone una segunda alternativa de estimación de la relación entre el crecimiento y las emisiones de $\mathrm{CO}_{2}$. Siguiendo a Mitic et al. (2017) se especifican los modelos de Dols y FMOLs para ambos grupos, considerando para el caso FMOLS una tendencia determinística en la relación de cointegración, mientras que esta no se considera en el caso de Dols debido a que la propia especificación incorpora el efecto dinámico en la relación entre las variables. En primer lugar, el estimador FMOLs propuesto por Phillip y Hansen (1990) surge de una transformación de la variable dependiente considerando las covarianzas asociadas a los residuos de los modelos en distintos periodos $t$, y de la primera diferencia temporal de los componentes determinísticos. Se 
encuentra que estos estimadores son asintóticamente insesgados y con distribución normal. En segundo lugar, el estimador Dols comparte la distribución asintótica de FMOLs, y se obtiene agregando al modelo adelantos de las diferencias de las variables determinística, incorporando efectos dinámicos (véase Wang \& Wu, 2012).

La tabla 5 muestra los resultados de los coeficientes estimados para FMOLS y DOLs. Según las estimaciones FMOLS, en el grupo 1 se verifica la EKC, encontrando que todos los coeficientes son significativos y con los signos esperados, en cambio en el grupo 2, si bien se obtienen los signos esperados según la hipótesis de la EKC, los coeficientes no resultan estadísticamente significativos. Para el caso de las estimaciones por el método DoLs, si bien también se obtienen coeficientes significativos, estos no tienen los signos esperados en ninguno de los dos grupos, no verificando la ЕKC. Se observa un efecto negativo del crecimiento económico en las emisiones de $\mathrm{CO}_{2}$, mientras que la variable al cuadrado tendría efecto positivo.

Tabla 5. Estimación: FMOLS y DOLS

\begin{tabular}{ccccc}
\hline & \multicolumn{2}{c}{ Grupo 1 } & \multicolumn{2}{c}{ Grupo 2 } \\
\cline { 2 - 5 } & FMOLS & DOLS & FMOLS & DOLS \\
\hline pib & $1,94^{* * *}$ & $-0,95^{* * *}$ & 0,71 & $-5,95^{* * *}$ \\
pib & $-0,07^{* * *}$ & $0,09^{* * *}$ & $-0,05$ & $0,27^{* * *}$ \\
gasto & $0,15^{* *}$ & $0,13^{* *}$ & $0,35^{* * *}$ & $0,44^{* * *}$ \\
apertura & $0,00^{* * *}$ & $-0,09^{* * *}$ & $-0.23^{* * *}$ & $-0,48^{* * *}$ \\
fbkf & $0,21^{* * *}$ & $0,31^{* * *}$ & $0,21^{* * *}$ & $0,31^{* * *}$ \\
\hline
\end{tabular}

Nota: ${ }^{*} 10 \% ; * * 5 \% ; * * 1 \%$.

Fuente: elaboración propia.

De esta forma, al considerar esta última estimación y el método FMOLs, el grupo con bajos niveles de emisiones verifica la EKC aludiendo a un aumento de las emisiones ante incrementos porcentuales de la actividad económica, hasta un punto de inflexión (turning point), después del cual un mayor nivel de producto interno bruto por habitante, reduce las emisiones de $\mathrm{CO}_{2}$ per cápita, logrando un crecimiento ambientalmente sostenible en el largo plazo. Considerando el método DoLs, incrementos en el producto real por habitante reduce el nivel de emisiones, aunque con indicios de que en el largo plazo con un producto real lo suficientemente alto, estas emisiones comiencen a incrementarse ante expansiones en la actividad económica. Estos resultados brindan una primera conclusión acerca de la virtud del uso de metodología 
de cluster previo a la estimación, debido a que se muestran comportamientos disímiles en base al grupo de países considerado. El omitir el estudio cualitativo previo, podría implicar en interpretaciones que no logren ajustarse de la mejor manera a todos los casos en estudio y que políticas mal formuladas en países en constante desarrollo conlleven a pérdidas en el bienestar económico sin lograr cometer el objetivo de un crecimiento sustentable.

\section{Conclusiones y consideraciones finales}

A partir de las series bidimensionales de emisiones de $\mathrm{CO}_{2} \mathrm{y}$ crecimiento económico, mediante el análisis de series temporales simbólicas y análisis de cluster, aplicados a 24 países de América Latina y el Caribe, se detecta la existencia de dos grupos de países con comportamientos diferenciados en términos de su dinámica de crecimiento económico y emisiones de $\mathrm{CO}_{2}$, que se diferencian por sus dispares niveles de emisiones durante el periodo considerado, no habiendo señales de potencial convergencia a largo plazo. El primer grupo se caracteriza por tener niveles reducidos de emisiones de $\mathrm{CO}_{2}$, está compuesto por 15 de los 24 países considerados en la muestra, pese a que muestran un crecimiento económico fluctuante a lo largo del periodo, comparativamente sus emisiones de $\mathrm{CO}_{2}$ en términos per cápita parecen reducidos. En oposición, el segundo grupo compuesto por otros 7 países muestran una dinámica con elevados niveles de emisiones de dióxido de carbono asociadas posiblemente a actividades de refinamiento de petróleo o un sector servicios (en particular, un sector turístico) desarrollado, el cual genera emisiones no solo fruto de la producción, sino también del consumo de combustible generado por el transporte. A estos dos grupos obtenidos, se suman Panamá y Uruguay que siguen cada uno un comportamiento propio, no integrándose a ninguno de los dos grupos obtenidos.

Se encuentran relaciones causales bidireccionales entre las emisiones de $\mathrm{CO}_{2}$ en el crecimiento económico, en ambos grupos. Adicionalmente se verifica la existencia de relaciones en el largo plazo. Esto se alinea a lo encontrado en la literatura de referencia tanto en los estudios de caso, como en aquellos que, al igual que este ejercicio, consideran un conjunto de países (Acheampong, 2018; Apergis \& Payne, 2015; Wang et al., 2017; Al Mulali et al., 2013). Luego de emplear diversos métodos de estimación, se encuentra que la verificación de la EKC depende del método de estimación empleado y del grupo considerado.

En el caso del grupo de bajas emisiones, para el cual las estimaciones por el método FMOLs sugieren la verificación de la EKC, un alternativa para este grupo de países puede ser continuar con un proceso productivo que genere 
crecimiento económico (a partir del desarrollo de actividades que no impliquen altos niveles de contaminación o por una focalización en el crecimiento a través del sector servicios) y, que de esta forma, la propia dinámica de crecimiento genere mejoras en el largo plazo en términos ambientales. Muchos de los trabajos de referencia en este ámbito se han esforzado en demostrar esta relación. Sin embargo, solo en algunos casos se logra verificar la hipótesis EKC (Sánchez \& Caballero, 2019; Balado-Neves et al., 2018; De Souza et al., 2018, Al Mamun et al., 2014) alineándose a los resultados para este subconjunto de países. Adicionalmente muchos de los países que integran este grupo, a lo largo del periodo han experimentado tasas de crecimiento del producto real per cápita por encima del promedio (permanencia en el régimen 2), por lo que se mostrarían capaces de generar crecimiento económico sostenible en el largo plazo. En el mediano plazo, aquellos casos donde aún el ingreso per cápita no sea suficiente para generar mejoras ambientales ante el crecimiento económico, sustituir el uso de energía por aquellas que no generen emisiones que contaminen el medio ambiente como el $\mathrm{CO}_{2}$, por ejemplo a partir de uso de energías renovables, parecería ser una alternativa de sustitución hacia una internalización de la polución y mitigación de los impactos negativos de las emisiones de carbono instalando así un tipo de crecimiento sostenible. Así mismo, políticas públicas que incentiven a dicha sustitución, por ejemplo, con apoyo a las empresas, e incentivando a cambios tecnológicos en el margen intensivo parece ser un posible camino a seguir. Sánchez-Caballero (2019) y Nguyen y Kakinaka (2018) validan en sus estudios estas conclusiones planteando la importancia de la sustitución hacia energías renovables y la mitigación del impacto negativo del crecimiento económico en la sostenibilidad ambiental.

En lo que respecta al grupo de altas emisiones, las estimaciones mediante EF y FMOLS no son significativas, por lo que no se encuentra evidencia a favor de la ЕKC; de modo que no sería plausible extraer conclusiones en términos e impacto y recomendaciones de política económica. Una posible explicación a lo anterior puede darse a partir de que el grupo de altas emisiones está conformado por poca cantidad de países, lo cual puede generar una pérdida de consistencia estadística de los resultados. Por otro lado, estos países con altas emisiones presentan dinámicas distintas entre ellos también (en la figura A1 se observa que tanto las emisiones como el crecimiento promedio presentan más variabilidad en el grupo de altas emisiones, respecto del de bajas emisiones). Por último, aunque la alternativa FMols logra su objetivo de dar robustez a la estimación por $\mathrm{EF}$, dado que se trata de paneles cointegrados, no se puede concluir lo mismo bajo la alternativa DoLs, ya que la relación encontrada se opone a la plantada por Kuznets. Sin embargo, la 
sustitución en el uso de energía hacia aquellas renovables que disminuyan la contaminación a partir de emisiones de $\mathrm{CO}_{2}$, parece como alternativa bajo contextos desfavorables (donde el crecimiento económico perjudique al medio ambiente).

En suma, se verifica la hipótesis preliminar de discrepancias en los comportamientos dinámicos de los países de la región en términos de crecimiento y emisiones, verificando la causalidad bidireccional para ambos grupos, pero con resultados en término de las relaciones entre las variables disímiles entre ellos, verificando solo en el caso de un grupo la EKc. Esto se condice con los resultados parciales encontrados en estudios como Al Mamun et al. (2014) y Narayan et al. (2015).

En primer lugar, si bien el agrupamiento del conjunto de países de la región, a la hora de cuantificar los efectos esperados tiene algunas ventajas, una limitación es que se cuenta con pocos países por grupo para la realización del análisis. Sin embargo, es claro también que la cantidad de países es limitada al tener como punto de partida un estudio regional. En segundo lugar, la simbolización de las series de crecimiento económico y emisiones de $\mathrm{CO}_{2}$ se utiliza como indicador de umbral a la media muestral anual, que suele ser muy sensible a datos extremos. No obstante, la metodología de simbolización propuesta permite la libre elección del indicador a utilizar, por tanto, se podrían considerar otros indicadores a fin de comparar resultados obtenidos; entre ellos puede trabajarse con la mediana muestral, cuartiles, o indicadores que no sean móviles al año, sino que sean fijos para todo el periodo. Por último, se puede destacar que la utilización del modelo lineal, así como da ventajas en términos de estimación e interpretación, es una limitante a la estructura dinámica de los datos, ya que a partir de la observación de los datos, las trayectorias permiten de forma más clara una dinámica no lineal en el tiempo.

Partiendo de lo anterior, algunos lineamientos a futuro pueden venir dados por la especificación de modelos que no tengan una dinámica lineal subyacente, sino que muestre otra especificación no lineal. Por un lado, a corto plazo puede desarrollarse alguna metodología que reemplace modelos estáticos, como en este caso, por modelos de paneles dinámicos que incluyan variables rezagadas y se ajusten de mejor forma a los procesos generadores de datos. Alternativas como el estimador de Arellano y Bond (1991) o ArellanoBover y Blundell y Bond (1995) puede que permitan desarrollar esta idea. Por otro lado, también se podría hacer el esfuerzo de incorporar al análisis los tratados y protocolos regionales e internacionales contra la mitigación de las emisiones de $\mathrm{CO}_{2}$, observando su efectividad no solo en niveles, sino también la existencia de cambio estructural en la región. 


\section{Agradecimientos}

Los autores agradecen al "Proyecto csic - Dinámica Económica”, Universidad de la República (UdelaR), Uruguay, por el financiamiento para la ejecución de este trabajo.

\section{Referencias}

Acheampong, A. (2018). Economic growth, $\mathrm{CO}_{2}$ emissions and energy consumption: What causes what and where?. Energy Economics, 74, 677-692. https://doi.org/10.1016/j.eneco.2018.07.022

Agbanike, T., Nwani, C., Uzuazie, U., Anochiwa, L., Onoja, T., \& Ogbonnaya, T. (2019). Oil price, energy consumption and carbon dioxide $\left(\mathrm{CO}_{2}\right)$ emissions: insight into sustainability challenges in Venezuela. Latin American Economic Review, 28(8), 1-26. https://doi.org/10.1186/s40503-019-0070-8

Al Mamun, Md., Sohag, K., Hannan Mia, Md.A., Uddin, G., \& Ozturk, I. (2014). Regional differences in the dynamic linkage between $\mathrm{CO}_{2}$ emissions, sectoral output and economic growth. Renewable and Sustainable Energy Reviews, 38, 1-11. https://doi.org/10.1016/j.rser.2014.05.091

Al Mulali, U. (2014). Investigating the impact of nuclear energy consumption on GDP growth and $\mathrm{CO}_{2}$ emission: A panel data analysis. Progress in $\mathrm{Nu}$ clear Energy, 73, 172-178. https://doi.org/10.1016/j.pnucene.2014.02.002

Al Mulali, U., Lee, J., Mohammed, A., \& Sheau-Ting, L. (2013). Examining the link between energy consumption, carbon dioxideemission, and economic growth in Latin America and the Caribbean. Renewable and Sustainable Energy Reviews, 26, 42-48. https://doi.org/10.1016/j.rser.2013.05.041

Al Mulali, U., Tang, CF., \& Ozturk, I. (2015). Estimating the Environmental Kuznets Curve hypothesis: evidence from Latin America and the Caribbean countries. Renewable and Sustainable Energy Reviews, 50, 918-925. https://doi.org/10.1016/j.rser.2015.05.017

Amin, A., Aziz, B., \& Liu, XH. (2020). The relationship between urbanization, technology innovation, trade openness, and $\mathrm{CO}_{2}$ emissions: evidence from a panel of Asian countries. Environmental Science and Pollution Research International, 27(28), 35349-35363. https://doi.org/10.1007/ s11356-020-09777-y

Angulo, A. (2010). Relación Entre Crecimiento Económico y Medio Ambiente: La U Ambiental De Kuznets. DELOS: Desarrollo Local Sostenible, 3(8), 4.

Antonakakis, N., Chatziantoniou, I., \& Filis, G. (2017). Energy consumption, $\mathrm{CO}_{2}$ emissions, and economic growth: An ethical dilemma. Renewable and Sustainable Energy Reviews, 68, 808-824. https://doi.org/10.1016/j. rser.2016.09.105 
Apergis, N., \& Payne, J.E. (2009). $\mathrm{CO}_{2}$ emissions, energy usage, and output in Central America. Energy Policy, 37, 3282-3286. https://doi.org/10.1016/j. enpol.2009.03.048

Apergis, N., \& Payne, J.E. (2014). Renewable energy, output, $\mathrm{CO}_{2}$ emissions, and fossil fuel prices in Central America: Evidence from a nonlinear panel smooth transition vector error correction model. Energy Economics, 42, 226-252. https://doi.org/10.1016/j.eneco.2014.01.003

Apergis, N., \& Payne, J.E. (2015). Renewable Energy, Output, Carbon Dioxide Emissions, and Oil Prices: Evidence from South America. Energy Sources, Part B: Economics, Planning, and Policy, 10(3), 281-287. https://doi.org/10. 1080/15567249.2013.853713

Arellano, M., \& Bond, S. (1991). Some tests of specification for panel data: Monte Carlo evidence and an application to employment equations. The Review of Economic Studies, 58(2), 277-297. https://doi.org/10.2307/2297968 Arellano, M., \& Bover, O. (1995). Another look at the instrumental variable estimation of error-components models. Journal of Econometrics, 68(1), 29-51. https://doi.org/10.1016/0304-4076(94)01642-D

Atta Mills, E. F. E., Zeng, K., \& Baafi, M. A. (2020). The economy-energyenvironment Nexus in IMF's Top 2 biggest economies: a TY approach. Journal of Business Economics and Management, 21(1), 1-22. https://doi. org/10.3846/jbem.2019.11321

Azam, M. (2016). Does environmental degradation shackle economic growth? A panel data investigation on 11 Asian countries. Renewable and Sustainable Energy Reviews, 65, 175-182. https://doi.org/10.1016/j.rser.2016.06.087

Balado-Naves, R., Baños-Pino, J.F., \& Mayor, M. (2018). Do countries influence neighbouring pollution? A special analysis of the EKC for $\mathrm{CO}_{2}$ emissions. Energy Policy, 123, 266-279. https://doi.org/10.1016/j.enpol.2018.08.059

Ben Jebli, M., \& Ben Youssef, S. (2016). Combustible renewables and waste consumption, agriculture, $\mathrm{CO}_{2}$ emissions and economic growth in Brazil. Carbon Management, 10(3), 309-321. https://doi.org/10.1080/175830 04.2019.1605482

Ben Jebli, M., Ben Youssef, S., \& Apergis, N. (2019). The dynamic linkage between renewable energy, tourism, $\mathrm{CO}_{2}$ emissions, economic growth, foreign direct investment, and trade. Latin American Economic Review, 28(1), 1-19. https://doi.org/10.1186/s40503-019-0063-7

Ben Mbarek, M., Saidi, K., \& Rahman, M.M. (2018). Renewable and nonrenewable energy consumption, environmental degradation, and economic growth in Tunisia. Quality E Quantity, 52(3), 1105-1119. https:// doi.org/10.1007/s11135-017-0506-7 
Boden, T. A., Anders, R. J., \& Marland, G. (2017). Global, Regional, and National Fossil-Fuel $\mathrm{CO}_{2}$ Emissions. Carbon Dioxide Information Analysis Center. https://doi.org/10.3334/CDIAC/00001_V2017.

Brida, J.G., London, S., \& Rojas, M. (2013). Desempeño económico regional: un análisis dinámico para el caso chileno en el periodo 1960-2009. Revista de Economía del Rosario, 16(2), 35-66. https://www.redalyc.org/ pdf/5095/509555097002.pdf

Chang, C.P., Dong, M., Sui, B., \& Chu, Y. (2019). Driving forces of global carbon emissions: from time- and spatial-dynamic perspectives. Economic Modelling, 77, 70-80. https://doi.org/10.1016/j.econmod.2019.01.021

Daw, C. S., Finney, C. E. A., \& Tracy, E. R. (2003). A review of symbolic analysis of experimental data. Review of Scientific Instruments, 74(2), 915-930. https://doi.org/10.1063/1.1531823

De Souza, E. S., Freire, F. de S., \& Pires, J. (2018). Determinants of $\mathrm{CO}_{2}$ emissions in the Mercosur: the role of economic growth, and renewable and nonrenewable energy. Environmental Science and Pollution Research, 25(21), 20769-20781. https://doi.org/10.1007/s11356-018-2231-8

Devire, S.A., \& Deviren, B. (2016). The relationship between carbon dioxide emisión and economic growth: hierarchical structure methods. Physica A: Statistical Mechanics and its Applications, 451(1), 429-439. https://doi. org/10.1016/j.physa.2016.01.085

Dumitrescu, E., \& Hurlin, C. (2002). Testing of Granger non-causality in heterogeneous panels. Economic Modelling, 29(4), 1450-1460. https://doi. org/10.1016/j.econmod.2012.02.014

Dutta, C., \& Das, D. (2016). Does disaggregated $\mathrm{CO}_{2}$ emission matter for growth? Evidence from thirty countries. Renewable and Sustainable Energy Reviews, 66, 825-833. https://doi.org/10.1016/j.rser.2016.08.034

Galeotti, M., Manera, M., \& Lanza, A. (2009). On the robustness of robustness checks of the environmental Kuznets curve hypothesis. Environmental and Resource Economics, 42(4), 551. https://link.springer.com/content/ pdf/10.1007/s10640-008-9224-x.pdf

Gardiner, R., \& Hajek, P. (2020). Interactions among energy consumption, $\mathrm{CO}_{2}$, and economic development in European Union countries. Sustainable Development, 28(4), 723-740. https://doi.org/10.1002/sd.2023

Gligo, N. (1993). Environment and natural resources in Latin American development. Development from Within. En O. Sunkel (Ed.), Toward a Neostrucuturalist Approach for Latin America. Lynne Rienner Publishers.

Gómez, M., \& Rodríguez, J.C. (2016). Analysis of causality between economic growth and carbon emissions: the case of Mexico 1971-2011. International 
Journal of Energy and Environmental Engineering, 10(12), 1086-1091. https:// doi.org/10.5281/zenodo.1128099

Grossman, G. M., \& Krueger, A. B. (1991). Environmental Impacts of a North American Free Trade Agreement. National Bureau of Economic Research. Working Paper, $\mathrm{n}^{\circ} 3914$.

Im, K.S., Pesaran, M.H., \& Shin, Y. (2003). Testing for unit roots in heterogeneous panels. Journal of Econometrics, 115(1), 53-74. https://doi. org/10.1016/S0304-4076(03)00092-7

Ito, $\mathrm{K}$. (2017). $\mathrm{CO}_{2}$ emissions, renewable and non-renewable energy consumption, and economic growth: Evidence from panel data for developing countries. International Economics, 151, 1-6. https://doi.org/10.1016/j. inteco.2017.02.001

Kantar, E., \& Keskin, M. (2013). The relationships between electricity consumption and GDP in Asian countries, using hierarchical structure methods. Physica A: Statistical Mechanics and its Applications, 392(22), 5678-5684. https://doi.org/10.1016/j.physa.2013.07.029

Kantar, E., Aslan, A., Deviren, B., \& Keskin, M. (2016). Hierarchical structure of the countries based on electricity consumption and economic growth. Physica A: Statistical Mechanics and its Applications, 454, 1-10. https://doi. org/10.1016/j.physa.2016.01.075

Kantar, E., Deviren, B., \& Aslan, A. (2019). Hierarchical structure of countries based on carbon dioxide emission over the periods of 1971-2012; the relationships economic growth and energy consumption. Chinese Journal of Physics, 60, 12-21. https://doi.org/10.1016/j.cjph.2019.02.008

Koengkan, M., \& Fuinhas, J.A. (2020). The interactions between renewable energy consumption and economic growth in the Mercosur countries. International Journal of Sustainable Energy, 39(6), 594-614. https://doi.org /10.1080/14786451.2020.1732978

Krackhardt, D., \& Stern, R.N. (1988). Informal networks and organizational crises: an experimental simulation. Social Psychology Quarterly, 51(2), 123-140. https://doi.org/10.2307/2786835

Kruskal, J.B. (1956). On the shortest spanning subtree of a graph and the traveling salesman problem. Proceedings of the American Mathematical Society, 7(1), 48-50. https://doi.org/10.2307/2033241

Le HP., \& Van, DTB. (2020). The energy consumption structure and African EMDEs' sustainable development. Heliyon, 6(4). https://doi.org/10.1016/j. heliyon.2020.e03822

Lean, H. H., \& Smyth, R. (2010). $\mathrm{CO}_{2}$ emissions, electricity consumption and output in ASEan. Applied Energy, 87(6), 1858-1864. https://doi. org/10.1016/j.apenergy.2010.02.003 
Levin, A., Lin, C.F., \& Chu, C. (2002). Unit root tests in panel data: asymptotic and finite-sample properties. Journal of Econometrics, 108(1), 1-24. https://doi.org/10.1016/S0304-4076(01)00098-7

López, L., \& Weber, S. (2017). Testing for Granger causality. The Stata Journal, 17(4), 972-984. https://doi.org/10.1177/1536867X1801700412

Mantegna, R.N., \& Stanley, H. (2000). An introduction to Econophysics: Correlations and Complexity in Finance. Cambridge University Press.

Mantegna, R.N. (1999). Hierarchical structure in financial markets. The European Physical Journal B-Condensed Matter and Complex Systems, 11(1), 193-197. https://doi.org/10.1007/s100510050929

Molgedey, L., \& Ebeling, W. (2000). Local Order, Entropy and Predictability of Financial Time Series. The European Physical Journal B-Condensed Matter and Complex Systems, 15(4), 733-737. https://doi.org/10.1007/s100510051178

Munir, Q., Lean, H. H., \& Smyth, R. (2020). $\mathrm{CO}_{2}$ emissions, energy consumption and economic growth in the ASEAN- 5 countries: a crosssectional dependence approach. Energy Economics, 85, 104571. https://doi.org/10.1016/j. eneco.2019.104571

Mitic, P., Munitlak Ivanovic, O., \& Zdravkovic, A. (2017). A cointegration análisis of real GDP and $\mathrm{CO}_{2}$ emissions in transitional countries. Sustainability, 9(4), 568. https://doi.org/10.3390/su9040568

Narayan, P.K., Saboori, B., \& Soleymani, A. (2015). Economic growth and carbon emissions. Economic Modelling, 53, 388-397. https://doi.org/10.1016/j. econmod.2015.10.027

Nguyen, KH., \& Kakinaka, M. (2018). Renewable energy consumption, carbon emissions, and development stages: some evidence from panel cointegration analysis. Renewable Energy, 132, 1049-1057. https://doi. org/10.1016/j.renene.2018.08.069

Ozturk, I. (2017). Measuring the impact of alternative and nuclear energy consumption, carbon dioxide emissions and oil rents on specific growth factors in the panel of Latin American countries. Progress in Nuclear Energy, 100, 71-81. https://doi.org/10.1016/j.pnucene.2017.05.030

Pao, H.T., \& Tsai, C.M. (2015). Modeling and forecasting the $\mathrm{CO}_{2}$ emissions, energy consumption, and economic growth in Brazil. Energy, 36(5), 2450-2458. https://doi.org/10.1016/j.energy.2011.01.032

Patiño, L., Padilla, E., Alcantara, V., \& Raymond, J.L. (2019). The relation of GDP per capita with energy and $\mathrm{CO}_{2}$ emissions in Colombia. Departmen d'Economia Aplicada. Facultar d'Economia i Empresa. Universitat Autónoma de Barcelona. 
Pedroni, P. (1999). Critical values for cointegration tests in heterogeneous panel with multiple regressors. Oxford Bulletin of Economics and Statistics, 61(S1), 653-670. https://doi.org/10.1111/1468-0084.0610s1653

Pedroni, P. (2001). Fully Modified ols for heterogeneous cointegrated panels. En B. H. Balragi, T.B. Fomby \& R. Carter Hill (Eds.), Nonstationary Panels, Panel Cointegration and Dynamic Panels (vol. 15) (pp. 93-130). Emerald Group Publishing Limited. https://doi.org/10.1016/S07319053(00)15004-2

Phillips, P. C. B., \& Hansen., B.E. (1990). Statistical inference in instrumental variables regression with I (1) processes. The Review of Economic Studies, 57(1), 99-125. https://doi.org/10.2307/2297545

Piccardi, C. (2004). On the control of chaotic systems via symbolic time series analysis. Chaos: An Interdisciplinary Journal of Nonlinear Science, 14(4), 1026-1034. https://doi.org/10.1063/1.1796071

Risso, W.A. (2019). Symbolic time series analysis and its application in social sciences. Time Series Analysis and Applications. Time Series Analysis and Applications. Zagreb. Croatia: InTech, 107-126. https://doi.org/10.5772/ intechopen.70826

Rodríguez-Caballero, C.V., \& Ventosa-Santaularia, D. (2017). Energy-growth long-term relationship under structural breaks. Evidence from Canada, 17 Latin American economies and the UsA. Energy Economics, 61, 121-134.

Saboori, B., Sulaiman, J., \& Mohd, S. (2012). Economic growth and $\mathrm{CO}_{2}$ emissions in Malaysia: a cointegration analysis of the environmental Kuznets curve. Energy Policy, 51, 184-191. https://doi.org/10.1016/j.enpol.2012.08.065

Saidi, S., \& Hammami, S. (2017). Modeling the causal linkages between transport, economic growth and environmental degradation for 75 countries. Transportation Research Part D: Transport and Environment, 53, 415-427. https://doi.org/10.1016/j.trd.2017.04.031

Salazar-Nuñez H., Vanegas-Martínez F., \& Tinoco-Zermeño M. (2020). Impact of energy consumption and carbon dioxide emissions on economic growth: Cointegrated panel data in 79 countries grouped by income level. International Journal of Energy Economics and Policy, 10(2), 218-226. ttps://doi.org/10.32479/ijeep.8783

Sánchez, L., \& Caballero, K. (2019). La curva de Kuznets Ambiental y su relación con el cambio climático en América Latina y el Caribe: un análisis de cointegración con panel, 1980-2015. Revista de Economía de Rosario, 22(1), 101-142. https://doi.org/10.32479/ijeep.8783

Santana de Souza, E., De Souza Freire, F., \& Pires, J. (2018). Determinants of $\mathrm{CO}_{2}$ emissions in the Mercosur: the role of economic growth, and 
renewable and non-renewable energy. Enviromental Science and Pollution Research, 25(21), 20769-20781. https://doi.org/10.1007/s11356-018-2231-8 Shahbaz, M., Ozturk, I., Afza, T., \& Ali, A. (2013). Revisiting the environmental Kuznets curve in a global economy. Renewable and Sustainable Energy Reviews, 25, 494-502. https://doi.org/10.1016/j.rser.2013.05.021

Souza Mendonca, A.K., De Andrade Conradi Barni, G., Moro, MF., Bornia, AC., Kupek, E., \& Fernandez, L. (2020). Hierarchical modeling of the 50 largest economies to verify the impact of GDP, population and renewable energy generation in $\mathrm{CO}_{2}$ emissions. Sustainable Production and Consumption, 22, 58-67. https://doi.org/10.1016/j.spc.2020.02.001

Sunkel, O., \& Gligo, N. (1980). Estilos de desarrollo y medio ambiente en América Latina (vol. 1). Fondo de Cultura Económica.

Tang, X.Z., Tracy, E.R., \& Brown, R. (1997). Symbol Statistics and SpatioTemporal Systems. Physica D: Nonlinear Phenomena, 102(3-4), 253-261. https://doi.org/10.1016/S0167-2789(96)00201-1

Vujović, T., Petković, Z., Pavlović, M., \& Jović, S. (2018). Economic growth based in carbon dioxide emission intensity. Physica A: Statistical Mechanics and its Applications, 506, 179-185. https://doi.org/10.1016/j.physa.2018.04.074

Wang, S., Li, G., \& Fang, C. (2017). Urbanization, economic growth, energy consumption, and $\mathrm{CO}_{2}$ emissions: Empirical evidence from countries with different income levels. Renewable and Sustainable Energy Reviews, 81, 2144-2159. https://doi.org/10.1016/j.rser.2017.06.025

Wang, Q., \& Wu, N. (2012). Long-run covariance and its applications in cointegration regression. The Stata Journal, 12(3), 512-542. https://doi. org/10.1177/1536867X1201200312

Wawrzyniak, D., \& Doryń, W. (2020) Does the quality of institutions modify the economic growth-carbon dioxide emissions nexus? Evidence from a group of emerging and developing countries. Economic research-Ekonomska Istraživanja, 33(1), 124-144.

Zafar, M., Mirza, F., Haider Zaidi, S., \& Hou, F. (2019). The nexus of renewable and nonrenewable energy consumption, trade openness, and $\mathrm{CO}_{2}$ emissions in the framework of ЕKC: evidence from emerging economies. Environmental Science and Pollution Research, 26(15), 15162-15173. https:// doi.org/10.1007/s11356-019-04912-w

Zambrano-Monserrate, M., Silva-Zambrano, C., Davalos-Penafiel, J., Zambrano-Monserrate, A., \& Ruano, MA. (2018). Testing envirnmental Kuznets curve hypothesis in Perú: The role of renewable electricity, petroleum and dry natural gas. Renewable and Sustainable Energy Reviews, 82, 4170-4178. https://doi.org/10.1016/j.rser.2017.11.005 
Zambrano-Monserrate, M., Troccoly-Quiroz, A., \& Pacheco-Borja, M.J. (2016). Testing the Environmental Kuznets Curve in Iceland: 1960-2010. Revista de Economía de Rosario, 19(1), 5-28. https://doi.org/10.12804/revistas. urosario.edu.co/economia/a.5239

\section{Apéndices}

Tabla A1. Proporción de permanencia en cada régimen (1951-2014)

\begin{tabular}{|c|c|c|c|c|c|}
\hline Código & País & $\mathrm{R}_{1}$ & $\mathrm{R}_{2}$ & $\mathrm{R}_{3}$ & $\mathrm{R}_{4}$ \\
\hline ARG & Argentina & $0 \%$ & $0 \%$ & $50 \%$ & $50 \%$ \\
\hline BRB & Barbados & $0 \%$ & $24 \%$ & $42 \%$ & $34 \%$ \\
\hline BOL & Bolivia & $59 \%$ & $41 \%$ & $0 \%$ & $0 \%$ \\
\hline BRA & Brasil & $39 \%$ & $58 \%$ & $0 \%$ & $3 \%$ \\
\hline $\mathrm{CHL}$ & Chile & $0 \%$ & $0 \%$ & $61 \%$ & $39 \%$ \\
\hline $\mathrm{COL}$ & Colombia & $28 \%$ & $54 \%$ & $9 \%$ & $9 \%$ \\
\hline CRI & Costa Rica & $36 \%$ & $64 \%$ & $0 \%$ & $0 \%$ \\
\hline CUB & Cuba & $0 \%$ & $1 \%$ & $55 \%$ & $44 \%$ \\
\hline DMA & Dominica & $34 \%$ & $66 \%$ & $0 \%$ & $0 \%$ \\
\hline DOM & República Dominicana & $36 \%$ & $50 \%$ & $13 \%$ & $1 \%$ \\
\hline ECU & Ecuador & $36 \%$ & $36 \%$ & $17 \%$ & $11 \%$ \\
\hline ESA & El Salvador & $56 \%$ & $44 \%$ & $0 \%$ & $0 \%$ \\
\hline GTM & Guatemala & $64 \%$ & $36 \%$ & $0 \%$ & $0 \%$ \\
\hline HTI & Haití & $74 \%$ & $26 \%$ & $0 \%$ & $0 \%$ \\
\hline HND & Honduras & $66 \%$ & $34 \%$ & $0 \%$ & $0 \%$ \\
\hline JAM & Jamaica & $1 \%$ & $13 \%$ & $31 \%$ & $55 \%$ \\
\hline MEX & México & $0 \%$ & $0 \%$ & $59 \%$ & $41 \%$ \\
\hline NIC & Nicaragua & $56 \%$ & $44 \%$ & $0 \%$ & $0 \%$ \\
\hline PAN & Panamá & $20 \%$ & $49 \%$ & $17 \%$ & $14 \%$ \\
\hline PRY & Paraguay & $56 \%$ & $44 \%$ & $0 \%$ & $0 \%$ \\
\hline PER & Perú & $41 \%$ & $59 \%$ & $0 \%$ & $0 \%$ \\
\hline LCA & Santa Lucía & $41 \%$ & $55 \%$ & $0 \%$ & $4 \%$ \\
\hline URY & Uruguay & $17 \%$ & $30 \%$ & $22 \%$ & $31 \%$ \\
\hline VEN & Venezuela & $0 \%$ & $0 \%$ & $47 \%$ & $53 \%$ \\
\hline
\end{tabular}

Fuente: elaboración propia. 


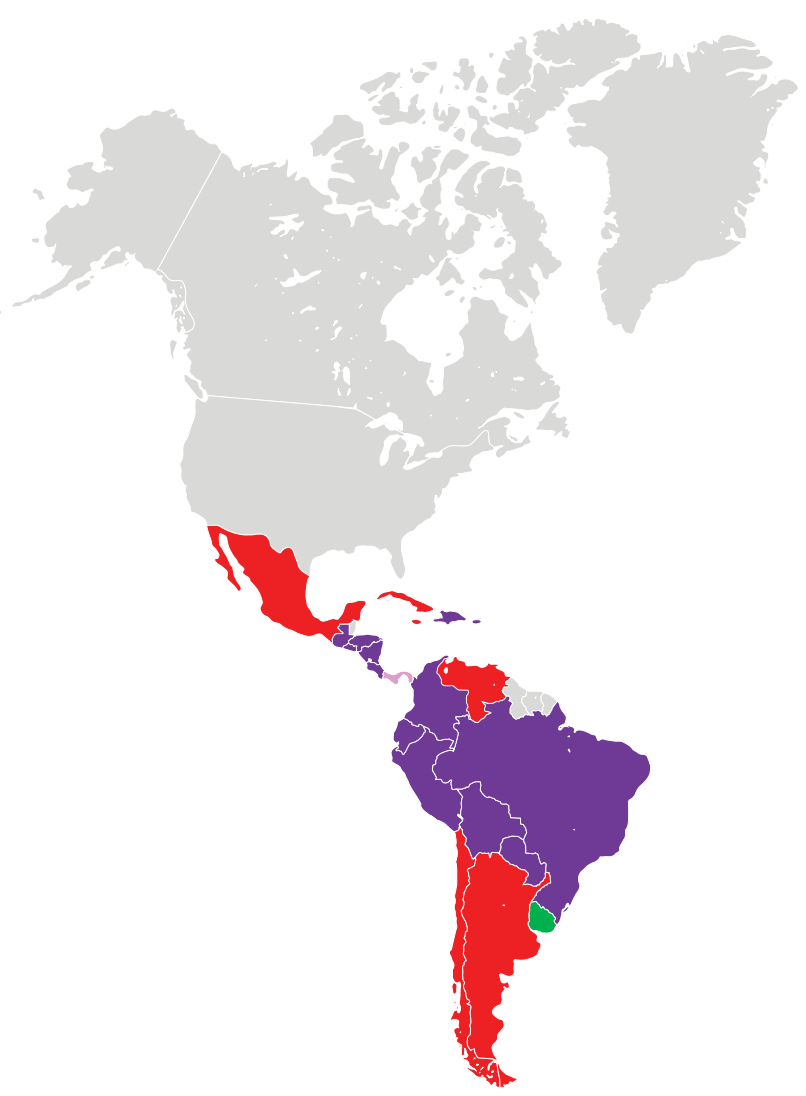

Figura A1. Distribución geográfica de los grupos (1951-2014)

Nota: Rojo: Grupo 1 (altas emisiones); Violeta: Grupo 2 (bajas emisiones), Verde: Uruguay; Rosado: Panamá; Gris: países que no integran la muestra.

Fuente: elaboración propia.

Tabla A2. Resumen descriptivo: emisiones de $\mathrm{CO}_{2}$ per cápita y tasa de crecimiento del PIB real per cápita (1951-2014)

\begin{tabular}{ccccccccc}
\hline & \multicolumn{3}{c}{ Emisiones de $\mathrm{CO}_{2}$ por habitante } & \multicolumn{5}{c}{ Tasa de crecimiento del PIB real por habitante } \\
\cline { 2 - 9 } & Media & Mediana & Mínimo & Máximo & Media & Mediana & Mínimo & Máximo \\
\hline Grupo 1 & 0,25 & 0,21 & 0,01 & 0,77 & 1,76 & 2,01 & $-28,6$ & 16,2 \\
Grupo 2 & 0,93 & 0,90 & 0,1 & 2,82 & 1,79 & 2,19 & $-23,2$ & 16,3 \\
Panamá & 0,43 & 0,44 & 0,17 & 0,77 & 3,08 & 3,11 & $-11,89$ & 12,74 \\
Uruguay & 0,48 & 0,47 & 0,29 & 0,70 & 1,79 & 2,58 & $-9,99$ & 9,20 \\
$\begin{array}{c}\text { Total } \\
\text { Países }\end{array}$ & 0,46 & 0,34 & 0,01 & 2,82 & 1,82 & 2,10 & $-28,6$ & 16,3 \\
\hline
\end{tabular}

Fuente: elaboración propia. 

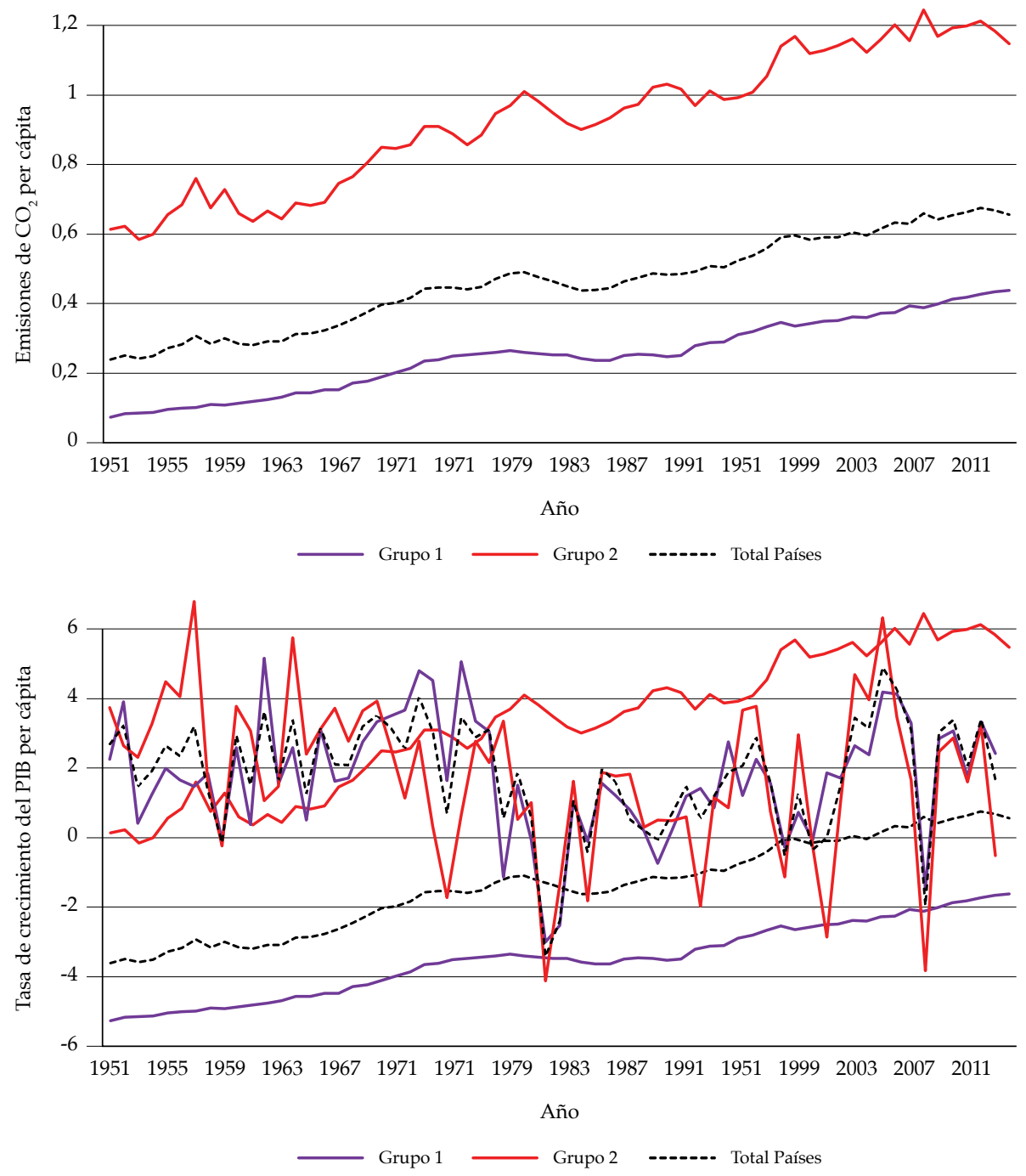

Figura A2. Trayectorias promedio de emisiones de $\mathrm{CO}_{2}$ per cápita y tasa de crecimiento del PIв real per cápita (1950-2014)

Fuente: elaboración propia. 
Total Países

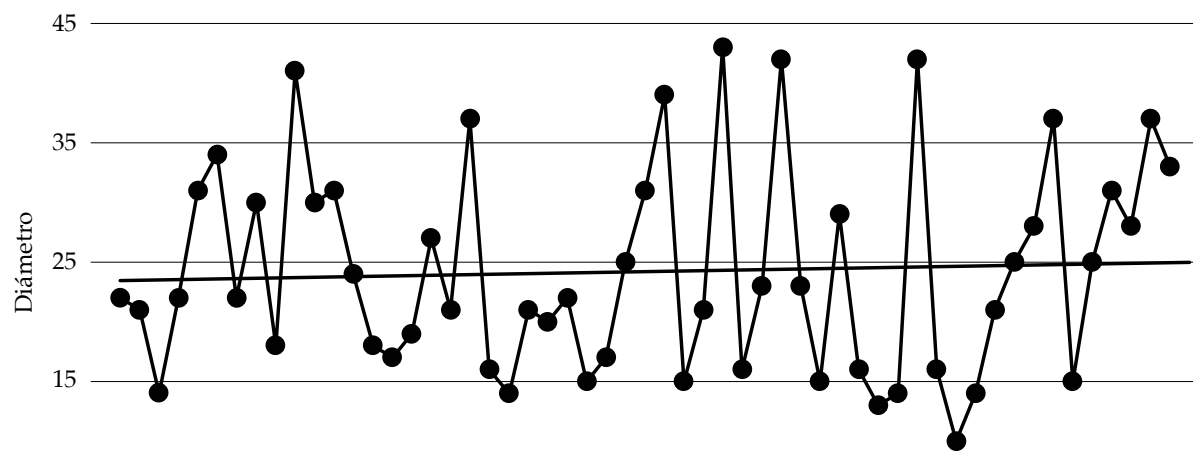

5

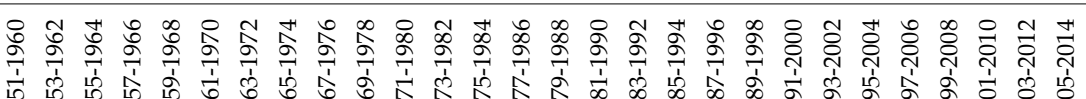

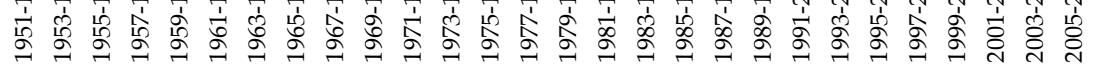
Ventana Temporal

Total Países

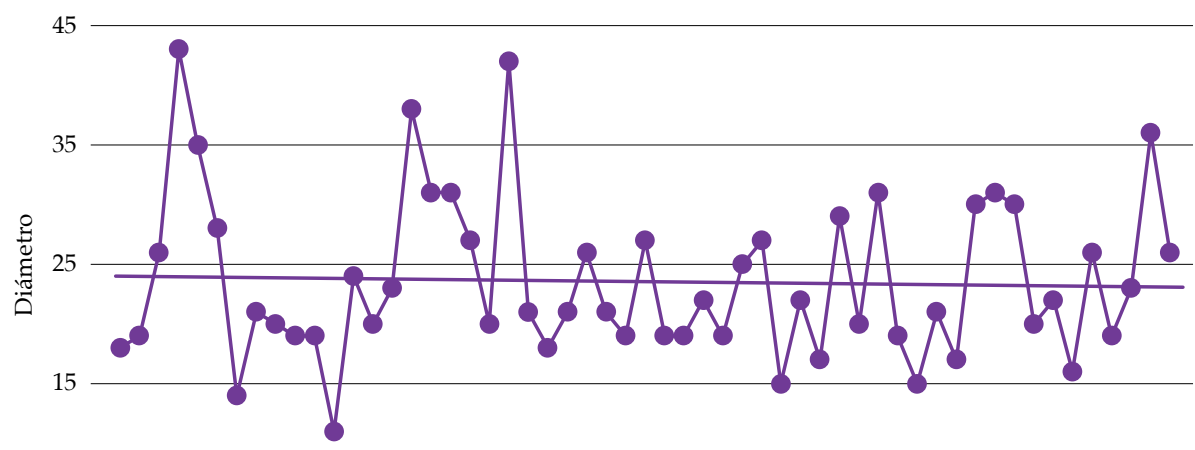

5

ஓ సু

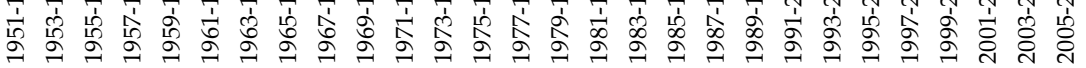
Ventana Temporal 
45

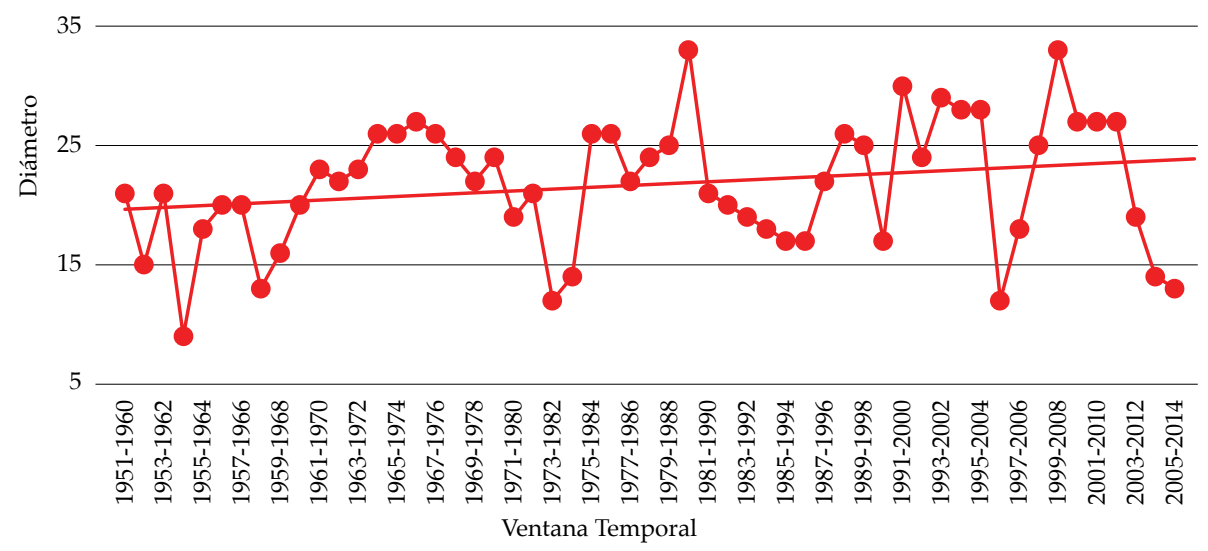

Figura A3. Evolución del diámetro de MST. Ventanas de 10 años

Fuente: elaboración propia.

Apartado A1. Test de raíces unitarias (Levin et al., 2002; Im et al., 2003)

Previo al análisis cuantitativo de los países de América Latina y el Caribe para la verificación de la EKC, es necesario precisar el orden de integración asociado a los procesos de cada uno de los indicadores considerados para dar validez a las potenciales relaciones causales encontradas y no caer en el análisis que sean espurias. Para ello, se emplearán dos test de raíces unitarias para paneles. La prueba de raíz unitaria para paneles de Levin-Lin y Chu (2002) se caracteriza por plantar una hipótesis nula de la existencia de al menos una raíz unitaria en el proceso analizado contra una hipótesis alternativa de paneles estacionarios. La característica central de esta prueba es que considera que el modelo subyacente del cual proviene el test es común entre todas las unidades del panel, tal como se puede observar en la especificación teórica de la ecuación (1).

$$
\Delta y_{i t}=\varphi y_{i, t-1}+z_{i t} \gamma_{i}+\sum_{j=1}^{P} \theta_{i j} \Delta y_{i, t-j}+\mu_{i t}
$$

El ejercicio consta en una prueba individual del parámetro $\varphi$ bajo la hipótesis nula de que el proceso no es estacionario y utiliza un estadístico $t$-ajustado con distribución asintóticamente normal. Por tanto, rechazar la hipótesis nula data de un proceso estacionario. 
Adicionalmente, se estudiará el orden de integración de los procesos considerados a partir de la prueba propuesta por Im, Pesarán y Shin (2003), que hace una nueva versión de lo anterior, dando la flexibilidad de considerar diferentes estructuras paramétricas dentro de las unidades del panel y un mejor ajuste a las conclusiones halladas. La ecuación (2) muestra analíticamente esta alternativa.

$$
\Delta y_{i t}=\varphi_{i} y_{i, t-1}+z_{i t} \gamma_{i}+\mu_{i t}
$$

En este caso se testea (nuevamente sobre los parámetros $\varphi_{i}$ ) la hipótesis nula de presencia de raíces unitarias en todo el panel contra la alternativa que algunos paneles son estacionarios.

Tabla A3. Pruebas de raíces unitarias

\begin{tabular}{ccccc}
\hline & \multicolumn{2}{c}{ Grupo 1 } & \multicolumn{2}{c}{ Grupo 2 } \\
\cline { 2 - 5 } & Levin-Lin y Chu & $\begin{array}{c}\text { Im, Pesaran } \\
\text { y Shin }\end{array}$ & Levin-Lin y Chu & $\begin{array}{c}\text { Im, Pesaran } \\
\text { y Shin }\end{array}$ \\
\hline $\log$ (pibpc) & 0,036 & 0,0246 & 0,7702 & 1,6130 \\
$\Delta \log$ (pibpc) & $-18,3129^{* * *}$ & $-19,8289^{* * *}$ & $-12,4589$ & $-12,3269^{* * *}$ \\
$\log$ (emisiones) & $-2,61267^{* *}$ & $-1,1421$ & $-0,8736$ & 1,0181 \\
$\Delta \log$ (emisiones) & $-33,7616^{* * *}$ & $-32,9813^{* * *}$ & $-15,3711$ & $-15,4837^{* * *}$ \\
$\log$ (gasto) & & $-1,2897^{*}$ & & $-1,7201$ \\
$\Delta \log$ (gasto) & & $-21,8137^{* * *}$ & & $-12,8756^{* * *}$ \\
$\log$ (apertura) & & $-0,7744$ & & $-0,2912$ \\
$\Delta \log$ (apertura) & & $-26,9679^{* * *}$ & & $-17,0020^{* * *}$ \\
$\log$ (fbkf) & $-2,0832^{* *}$ & $-1,1579$ & $-2,6642^{* * *}$ & $-2,1347^{* *}$ \\
$\Delta \log$ (fbkf) & $-19,0551^{* * *}$ & $-18.3575^{* * *}$ & $-12,0476^{* * *}$ & $-11,8112^{* * *}$ \\
\hline
\end{tabular}

Nota: *H0: el proceso contiene raíces unitarias; ${ }^{* * *} 1 \% ; * * 5 \% ;{ }^{*} 10 \%$.

Fuente: elaboración propia.

La tabla A3 resume los resultados de la aplicación de las pruebas de raíz unitaria para todas las variables. ${ }^{8}$ Como se puede observar, aunque no se rechaza la hipótesis nula para las variables en logaritmos al 1\%, sí se logra

8 La prueba de Levin-Li y Chu requiere de un panel completamente balanceado, por lo que no se puede realizar para las variables de gasto del Gobierno y apertura comercial internacional, por tener datos faltantes en las series. 
rechazar para cualquier nivel de confianza que la primera diferencia de ambas variables es estacionaria. Por lo tanto, todos los procesos son integrados de primer orden. 